Review

\title{
Multivariate Curve Resolution: 50 years addressing the mixture analysis problem - A review
}

\author{
Anna de Juan ${ }^{\mathrm{a},}{ }^{*}$, Romà Tauler ${ }^{\mathrm{b}}$ \\ ${ }^{a}$ Chemometrics Group. Universitat de Barcelona. Dept. of Chemical Engineering and Analytical Chemistry, Martí I Franquès, 1, 08028, Barcelona, Spain \\ ${ }^{\mathrm{b}}$ IDAEA-CSIC. Environmental Chemometrics Group. Department of Environmental Chemistry, Jordi Girona 18, 08034, Barcelona, Spain
}

\section{H I G H L I G H T S}

- The main advances of Multivariate Curve Resolution from 1971 to 2020 are reviewed.

- New constraints based on mathematical or natural profile properties are described.

- New challenging data structures used in MCR are presented.

- Main advances in estimating and understanding the ambiguity phenomenon are addressed.

- New application domains, such as -omics, imaging or multidimensional chromatography are mentioned.

\section{A R T I C L E I N F O}

\section{Article history:}

Received 25 August 2020

Received in revised form

21 October 2020

Accepted 25 October 2020

Available online $\mathrm{xx}$

\section{Keywords:}

Multivariate curve resolution Constraints

Ambiguity

Multiset analysis

Process analysis

Environmental data analysis

Hyperspectral image analysis

Multidimensional chromatography

-Omics data analysis

Big data

\section{G R A P H I C A L A B S T R A C T}

\section{MCR 50 years}

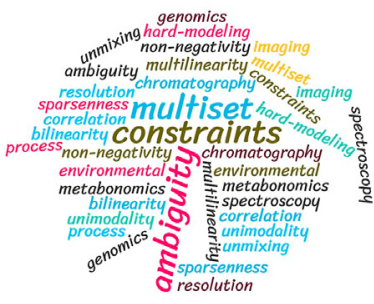

\begin{abstract}
A B S T R A C T
Multivariate Curve Resolution (MCR) covers a wide span of algorithms designed to tackle the mixture analysis problem by expressing the original data through a bilinear model of pure component meaningful contributions. Since the seminal work by Lawton and Sylvestre in 1971, MCR methods are dynamically evolving to adapt to a wealth of diverse and demanding scientific scenarios. To do so, essential concepts, such as basic constraints, have been revisited and new modeling tasks, mathematical properties and domain-specific information have been incorporated; the initial underlying bilinear model has evolved into a flexible framework where hybrid bilinear/multilinear models can coexist, the regular data structures have undergone a turn of the screw and incomplete multisets and matrix and tensor combinations can be now analyzed. Back to the fundamentals, the theoretical core of the MCR methodology is deeply understood due to the thorough studies about the ambiguity phenomenon. The adaptation of the method to new analytical measurements and scientific domains is continuous. At this point of the story, MCR can be considered a mature yet lively methodology, where many steps forward can still be taken.
\end{abstract}

(c) 2020 Elsevier B.V. All rights reserved.

\footnotetext{
* Corresponding author.

E-mail address: anna.dejuan@ub.edu (A. de Juan).
} 


\section{Contents}

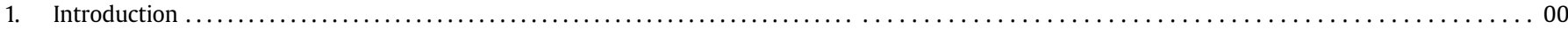

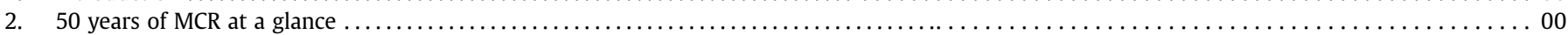

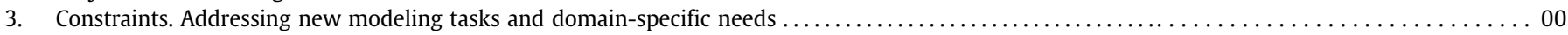

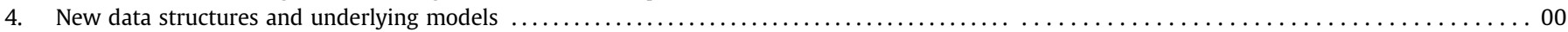

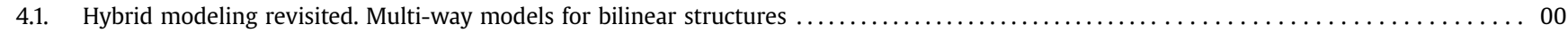

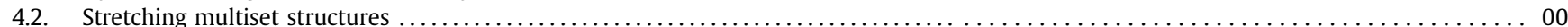

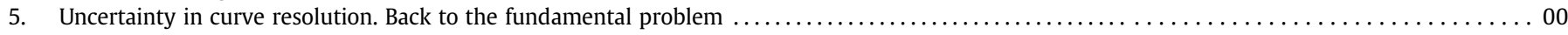

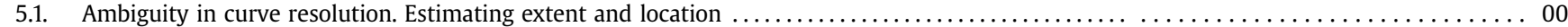

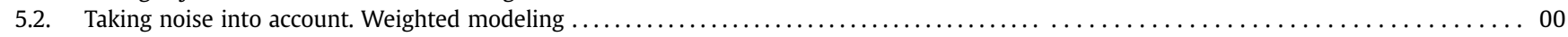

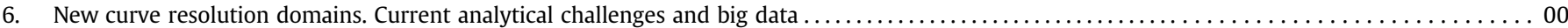

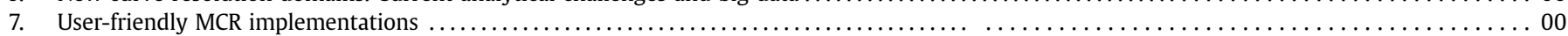

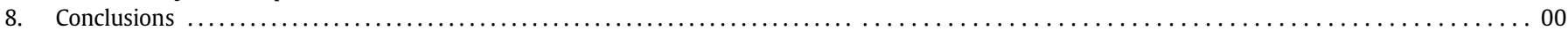

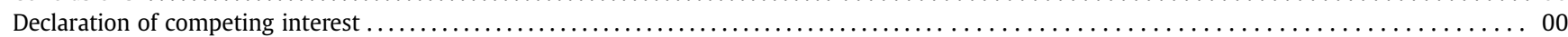

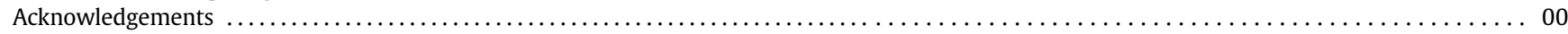

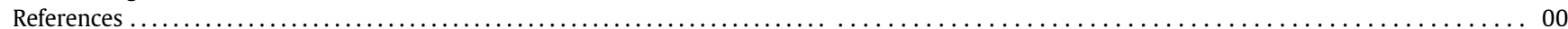

\section{Introduction}

The so-called mixture analysis problem has been a constant concern in any scientific domain since ever. Formulated in different ways, it comes down to realize that all what is observable or measurable in a real system seldom responds to a single cause or source. Variations in a collected response as a function of time, space, sample or in different experimental/environmental/clinical conditions, are the global expression of the contributions of a number of individual sources of variation. Understanding the nature and apportionment of these individual sources to the global and mixed available response is essential to understand the system under study.

How to formulate the mixture analysis model is a primary issue. When the response is an instrumental measurement, the individual signal contributions can be often described through an additive model. When the response is more complex, e.g., a set of environmental parameters defining an ensemble of sampling points, doubts can be casted upon the suitability of such a simple model. However, in the absence of a clear knowledge about the relationship among the individual response contributions, the principle of parsimony justifies the use of the simplest possible model to explain a phenomenon. Hence, Multivariate Curve Resolution methods address the mixture analysis problem using a bilinear model, or in cases of having multiway data its multilinear extension.

In general, in its simplest formulation, the responses collected as a function of any variable or set of conditions are organized in a data table $\mathbf{D}$, where each row is the global mixed response at a certain stage. Considering that the mixed responses in a data table D come from $n$ sources of variation, the mixture analysis model can be expressed as:

$\mathbf{D}=\sum_{\mathrm{i}=1}^{\mathrm{n}} \mathbf{c}_{\mathbf{i}} \mathbf{s}_{\mathbf{i}}^{\mathbf{T}}+\mathbf{E}$

where $\mathbf{c}_{\mathbf{i}}$ is the qualitative profile that describes the nature of the source of variation and is the related apportionment profile. $\mathbf{E}$ would stand for the variation unexplained by the model. In a more compact form, equation (1) turns into equation (2), which is the general expression of the bilinear MCR model in the linear algebra matrix form. The matrix $\mathbf{S}^{\mathbf{T}}$, with $n$ rows, would contain the qualitative profiles of the individual sources of variation, whereas the matrix $\mathbf{C}$, with $n$ columns, would contain the related apportionment profiles.

$\mathbf{D}=\mathbf{C S}^{\mathbf{T}}+\mathbf{E}$

The wideness of the mixture analysis problem is connected to the diversity of the concept of component. In general, a component is any entity that can present a qualitative distinct profiles $\mathbf{s}_{\mathbf{i}}^{\mathbf{T}}$, that remains invariant and valid to describe the information in $\mathbf{D}$. This distinct qualitative profile can be related to simple entities, e.g., the pure spectrum of a chemical compound, or to more complex entities, e.g., the spectral fingerprint of a biological tissue or the compositional profile of a contamination source. The profiles in matrix C, whichever their specific meaning, are defined as the apportionment (or concentration) profiles of each of the sources identified. Two paradigmatic examples of MCR applications and their related bilinear models are described next for further clarification. In the analysis of a chromatogram with UV detection, the data table (or matrix) D would be formed by the UV spectra collected as a function of the elution time, $\mathbf{S}^{\mathbf{T}}$ would contain the pure spectra of the eluted compounds and $\mathbf{C}$ the related concentration profiles (in this case, the chromatographic elution peaks). Likewise, in an environmental study monitoring several contaminants in a geographical area, the data table $\mathbf{D}$ would be formed by the concentrations of the different contaminants determined in each geographical sampling point, the matrix $\mathbf{S}^{\mathbf{T}}$ would contain the compositional profiles of each contamination source, showing the relative abundance of the measured contaminants, and the matrix $\mathbf{C}$ has the related geographical apportionment profiles.

Once put in context the general mixture analysis problem and the related MCR model, the next sections of this review will cover the evolution of this methodology, stressing key concepts linked to fundamental developments, impact in different application domains and providing friendly hands-on tools and graphical program interfaces that have brought this methodology to the general user.

\section{50 years of MCR at a glance}

Early 70's is the official date of birth of the methodologies coined as Curve Resolution methods. The seminal work by Lawton and Sylvestre in 1971 [1], entitled 'Self Modeling Curve Resolution' stated in the abstract: 'This paper presents a method for determining 
the shapes of two overlapping functions $f_{1}(x)$ and $f_{2}(x)$ from an observed set of additive mixtures, $\left\{\alpha_{i} f_{1}(x)+\beta_{i} f_{2}(x) ; i=1, \cdots, n\right\}$, of the two functions. This type of problem arises in the fields of spectrophotometry, chromatography, kinetic model building, and many others' and clarified in the introduction: The techniques described in this paper may be applied to any experiment whose outcome is a continuous curve $Y(X)$ which is an additive mixture of two unknown, nonnegative, linearly independent'. Although nowadays this definition may seem limited for many reasons, it was clearly pointing out several aspects repeated in all future MCR developments, namely: a) the usefulness of a bilinear model to describe the mixture analysis problem, b) the need to work with natural constraints, e.g., non-negativity, to adapt to the characteristics of chemical mixtures and recover the underlying profiles of the individual contributions, c) the soft-modeling nature of the method, since no model-based shapes were assumed for the profiles to be recovered and d) the diversity of problems that this methodology can tackle. Another relevant aspect pointed out in this seminal work was that there were a range of feasible solutions compatible with the sought bilinear model, which obeyed the natural preset constraints and provided an optimal reproduction of the original data set $\mathbf{D}$, i.e., the ambiguity concept was defined as the main source of uncertainty in any MCR method. It is not surprising that most research in the immediate years after this first work was oriented to increase the number of contributions and the complexity of the systems to be resolved and to understand the concept of ambiguity numerically and through graphical representations of feasible solution spaces, such as the Borgen plots [2].

The 80's decade was an intensive period of development of many of the MCR algorithms that we know nowadays. MCR was generally understood as the methodology providing a bilinear model formed by chemically meaningful profiles, but different approaches were proposed to recover the profiles sought. A common important basis in many of these methods was the use of the close connection between the bilinear decomposition provided by Principal Component Analysis (PCA) and the MCR model. Both PCA and MCR provide a bilinear description of the data set with maximum variance explained. In a noise-free scenario, this means that the space defined by PCA scores and concentration profiles (C) and the space defined by PCA loadings and response profiles $\left(\mathbf{S}^{\mathbf{T}}\right)$ are identical. Therefore, many algorithms used PCA as a prior step to the MCR analysis to estimate the number of components of the MCR model or worked finding the concentration and spectra profiles as rotations of scores and loadings, respectively. There were two main families of methods during this period: the non-iterative [3] and the iterative MCR algorithms [4]. The non-iterative MCR algorithms worked using a single calculation step to recover each particular component in the bilinear model. Most of them were using auxiliary local rank methods, such as Evolving Factor Analysis (EFA) or related methods [5-7], to set the concentration windows (i.e., D rows where a component existed) and the zeroconcentration windows (i.e., D rows where the component did not exist) of each component to be resolved. This task was possible because the niche of application of MCR methods was restricted to process-like data, where a sequential emergence-decay of the concentration profiles of components could be very often assumed. Knowing the concentration windows of all components, suitable D data subspaces could be built with special characteristics that properly combined provided directly either the concentration profile, e.g., in Window Factor Analysis (WFA) [8], or the pure response of a particular component, e.g., in Subwindow Factor Analysis (SFA) [9]. In this way, the $\mathbf{C}$ or $\mathbf{S}^{\mathbf{T}}$ profiles of all components were sequentially recovered. The counterpart matrix of the bilinear model could be easily recovered by a single least-squares step using the available $\mathbf{C}$ or $\mathbf{S}^{\mathbf{T}}$ matrix and the original data set $\mathbf{D}$. Instead, iterative resolution methods were working using initial estimates of $\mathbf{C}$ or $\mathbf{S}^{\mathbf{T}}$ profiles that were iteratively optimized under the action of constraints. This iterative optimization could take place in the $\mathbf{C}$ and/or the $\mathbf{S}^{\mathbf{T}}$ space and the profiles of components could be recovered sequentially or simultaneously. Initial estimates were usually derived from local rank analysis methods [5] or using methods based on pure variable detection [10,11]. Iterative Target Transformation Factor Analysis (ITTFA) was the first iterative method proposed independently by Gemperline [12] and Vandeginste [13]. Taking as a basis the score space, the initial target of a concentration profile was iteratively projected onto the score space and the projected profile corrected according to the constraints selected, e.g., non-negativity, until the difference between the corrected and projected target was low enough. Sequentially, all C profiles were recovered, and afterwards $\mathbf{S}^{\mathbf{T}}$ was recovered by leastsquares. Soon after ITTFA, the method doing a simultaneous Alternating Least Squares optimization of full $\mathbf{C}$ and $\mathbf{S}^{\mathbf{T}}$ matrices under constraints appeared $[4,14,15]$. This method, known nowadays as Multivariate Curve Resolution-Alternating Least Squares (MCR-ALS), worked with initial estimates of either the full matrix $\mathbf{C}$ or $\mathbf{S}^{\mathbf{T}}$ and, in every iteration, calculated both matrices of the bilinear model under the action of suitable constraints. When the reproduction of the original data set $\mathbf{D}$ by the model $\mathbf{C S}^{\mathbf{T}}$ was good enough, the optimization stopped. In the early formulation of this method, only work with a single data matrix was performed. MCRALS did not need the use of the scores or loading spaces to perform the optimization process but could work using a PCA-filtered D matrix as the reference for optimization if required.

The 90's decade milestone was the emergence of multiset analysis, very much linked to the MCR-ALS algorithm [16,17]. In this decade, the use of MCR algorithms designed to work specifically with a single data set started to decay due to the ambiguity problem, even if the necessary conditions for unique solutions, based on local rank and on the pattern of compound overlap, were very well stated by two fundamental resolution theorems proposed by Manne [18]. The different situations where unique solutions could be recovered without ambiguities using MCR-ALS with local rank and selectivity constraints or when three-way data were analyzed were examined in detail at the same time [19]. Multiset analysis allowed data fusion, i.e., the flexible use of many data subsets containing diverse information in a single data set structure. On one hand, multiset analysis provided a new world of possibilities in terms of the chemical problems that could be addressed, and, on the other hand, the amount of complementary information used significantly decreased the ambiguity of the final MCR solutions. Around the same period, multi-way analysis methods entered strongly in the chemical field [20,21], with algorithms such as PARAFAC [22,23] or Tucker methods [24,25], oriented also to handle several data subsets in tensor-like structures. The main differences between multiset analysis and multi-way analysis algorithms were the requirements of the initial data structure, with more modes in common in multi-way analysis than in multiset analysis and, most important, the underlying model, bilinear for multiset analysis or multilinear or with factor interaction in multiway analysis. Multi-way analysis based on methods like PARAFAC or Direct Trilinear Decomposition [26] offered the advantage of unique solutions, whereas multiset analysis still suffered from a certain degree of ambiguity but allowed the work with more flexible data set structures and a very versatile use of constraints. These two ways to analyze the data continue nowadays and are complementary. Around this period, MCR was no longer restricted to process-like data and entered strongly in other domains, such as the analysis of environmental data sets [27,28].

The 21 st century brought many additional advances. In the first decade, very advanced constraints showed up. Among those, hard- 
modeling constraints, which allowed implementing first principle models to provide defined shapes to concentration profiles [17,29-31], or the correlation constraint [4,32,33], which incorporated an inner calibration model in the MCR optimization. The obvious link between multiset and multi-way analysis also promoted the emergence of model constraints that allowed the basic MCR bilinear model turn into a multilinear [34,35] or factor interaction model $[17,36]$. Other than the inclusion of new constraints, the ambiguity problem was still a concern. Within this topic, several approaches appeared to help in the estimation of extent and location of ambiguity in the MCR solutions considering the effect of the different constraints. Two tendencies were explored around this topic: simpler methodologies oriented to provide maximum and minimum extreme values for the MCR solutions [37,38], easy to use and apt for systems with an unlimited number of components; and more complex approaches meant to span the whole range of feasible solutions, more complete to define this phenomenon, but limited to be used and visualized to a maximum of four-component systems [39,40]. The first decade of 2000 also opened MCR to new application domains linked to the emergence of complex analytical measurements, such as hyperspectral image analysis [41-43] and multidimensional chromatography [44-46].

The present decade has revisited the data structures and models in MCR $[17,20]$. New data structures, such as incomplete multisets, oriented to address the problem of missing blocks of information, have appeared with the related algorithm variant to handle them [47]. Adaptations to fuse originally 2D and 3D data arrays using dedicated hybrid bilinear/multilinear models have also been proposed [48]. Some of the new fields of application, such as hyperspectral image analysis, have seen the emergence of dedicated constraints incorporating spatial characteristics [49-51]. The fundamental studies on ambiguity continue in view of the new constraints and data structures born [39,40,52]. Finally, in line with the global trend of big data, MCR has entered in new domains characterized by the need to analyze massive amounts of information, such as the -omics world, which includes intrinsically strong challenges in terms of representative data compression and efficient data fusion [53,54].

Fig. 1 tries to summarize the most relevant milestones in MCR history. Many of the most recent developments of MCR will be developed in next sections. At this point, it is necessary to remember all authors that have provided their perspectives during the whole history of MCR. Their reviews on this topic may help to understand in more detail the past developments and the motivation for the ongoing research progress [55-60].

\section{Constraints. Addressing new modeling tasks and domain- specific needs}

A constraint can be generally defined as any systematic chemical or mathematical property obeyed by concentration and/or response profiles that can be encoded under the form of a mathematical condition included in an algorithm [4,17,60]. Constraints are the corner stone of iterative MCR methods, provide chemical meaning to the resolved profiles and, when properly applied, decrease or completely suppress the ambiguity associated with MCR solutions. Constraints, when properly applied, should not change significantly the model fit to the data. The initial constraints in MCR methods were linked to spectroscopically monitored process data sets and reflected simple chemical properties, such as non-negativity, unimodality (presence of one maximum per profile) or closure (mass balance condition), or relevant mathematical knowledge to decrease ambiguity, such as the selectivity or local rank constraints $[4,17,19,61,62]$. The proper use of these constraints provided very satisfactory results in chromatographic or reaction data. Since early times, the idea of selecting constraints differently per mode ( $\mathbf{C}$ and/or $\mathbf{S}^{\mathbf{T}}$ profiles), per component and per $\boldsymbol{C}$ or $\boldsymbol{S}^{\boldsymbol{T}}$ submatrix (in multiset analysis) conferred to MCR the possibility to become a tailor-made approach, adaptable to data set structures of very diverse typologies and chemical information $[4,17]$.

Other than the early constraints described above, the $\mathrm{XXI}^{\text {st }}$ century brought a different kind of constraints, based on modeling tasks typically associated with other data analysis methods, such as the hard-modeling constraint [17,29-31], closely related to chemical hard-modeling by non-linear parametric fitting, and the correlation constraint [4,32,33] linked to direct multivariate calibration methods. This new kind of constraints could be interpreted as a win-win solution, since they surmounted the main limitation linked to the data analysis methods they were associated with, i.e., the need to have full knowledge of the behavior of all signal contributions (components), and were providing MCR solutions with additional information beyond the plain soft-modeling

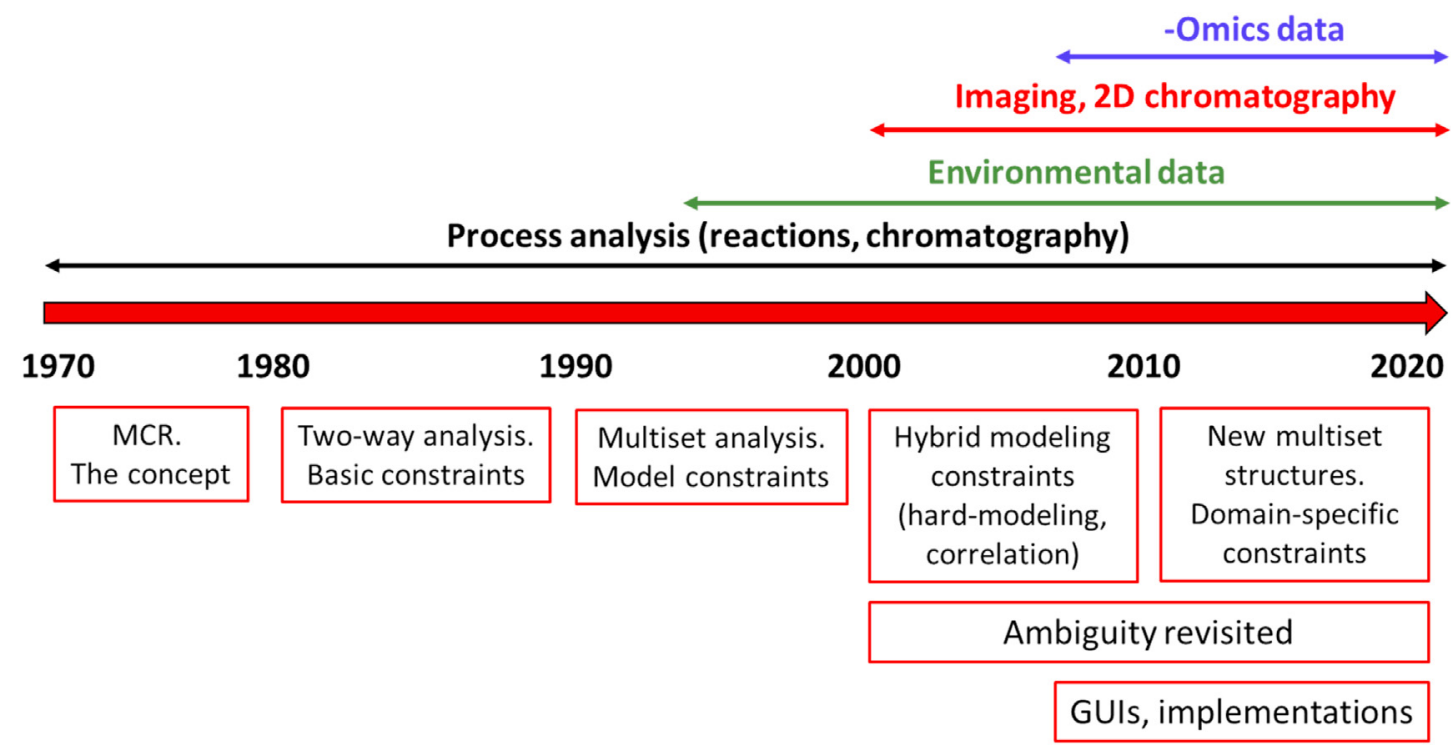

Fig. 1. Milestones in MCR history and main MCR application domains. 
output, namely fitted chemical parameters from the hard-modeling constraint or concentration profiles in real concentration units when the correlation constraint was applied. The per component and per block flexibility in the application of constraints enabled the possibility to use 'grey' models, i.e., models where some components or experiments could use explicitly this new hard-modeling or calibration information, whereas some others were simply modeled using other classical soft-modeling constraints.

To understand the value of the hard-modeling constraint, it is essential to realize the difference in the model fitting step between classical hard-modeling and the related constraint used in MCR. In pure hard-modeling, the variation of the initial experimental data (D) is directly fitted with the chemical hard-model and the residuals minimized are expressed as: $\min \left\|\mathbf{D}\left(\mathbf{I}-\mathbf{C C}^{+}\right)\right\|$. This implies that all signal variation recorded should respond to the preset model. In contrast, in the hard-modeling constraint in MCR-ALS, the fitting step is performed on the concentration profiles, C, i.e., in the unmixed information and, therefore, it is easy to select which components need to be involved in the model and which contributions can be left outside (see Fig. 2a). Such a specificity of MCRALS has allowed the possibility to fit a physicochemical model in the presence of spurious signal contributions that were not taking part in the process of interest [29]. These external contributions could be due to strong backgrounds, sample matrix absorption [63], or simply to other physical or chemical phenomena that were accompanying a process while it was taking place, such as conformational transitions [64] or scattering changes linked to kinetic reactions [65]. Hard-modeling constraints have been linked to

a)
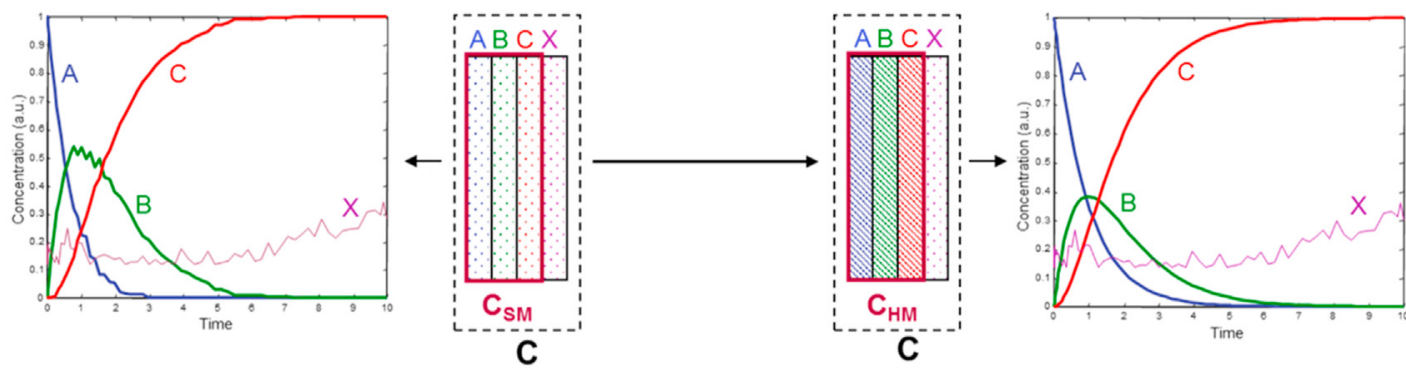

b)
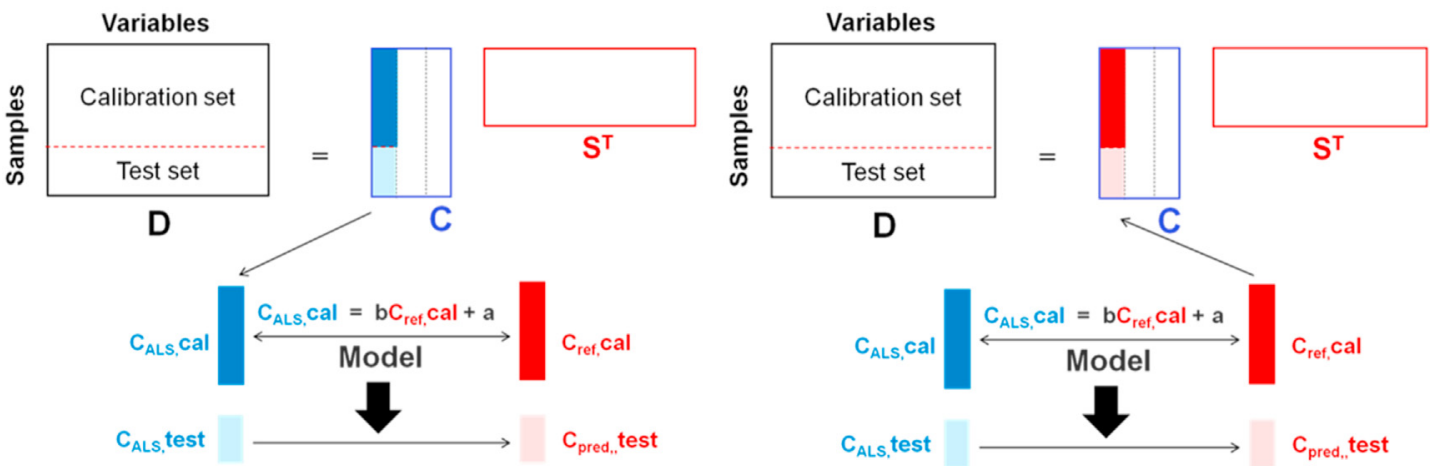

c)
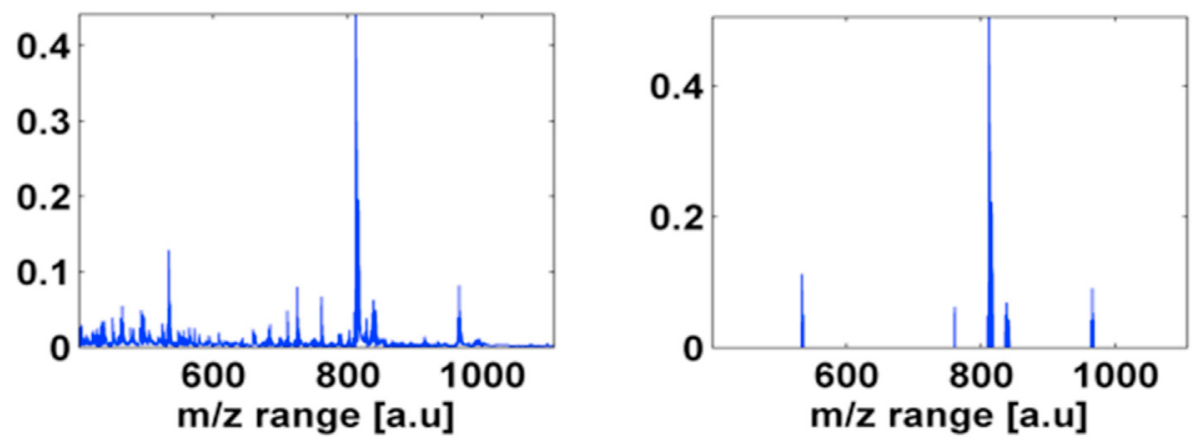

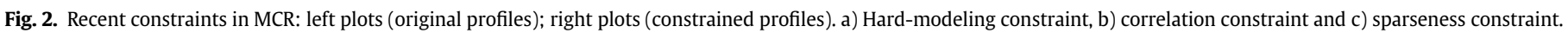


different kinds of physicochemical models, such as kinetic [29,64,65], equilibria [66], or enzymatic models [63], and they have served for process understanding through the interpretation of the related mechanisms and fitted parameters or for quantitative purposes, when one of the fitted parameters was a total concentration $[63,66]$. In photochemical processes, the use of hard-modeling constraints has served to deal with specific instrumental artifacts linked to ultrafast reaction monitoring and hard-models have been convoluted with instrumental response functions for a better performance [67]. The use of hard-modeling constraints in MCR-ALS has also helped to overcome typical limitations of soft-modeling methods, such as the impossibility to model non-absorbing species, since some of them can be linked to absorbing ones through the hard-model. Such a fact has been essential in the modeling of initial contributions in difference spectra $[64,68]$. When multiset analysis of several experiments is used, classical hard-modeling needs a model link between all experiments analyzed, i.e., a single global model or some equation that can link the models of different experiments. This requirement is not needed in the MCR framework, since the common $\mathbf{S}^{\mathbf{T}}$ matrix of the bilinear model works as the link within the multiset [29]. In the multiset context, the per block flexibility in the application of hard-modeling constraints to the $\mathbf{C}$ submatrices in a multiset allows working with a single global model including all experiments in this data structure, several global models for different experiment subsets and, most important and unique, allows combining model-based and modelfree experiments in the same multiset, such as in combinations of UV-based spectroscopic monitoring and HPLC-UV chromatographic data linked to the same chemical system [69,70].

From a mathematical and chemical point of view, the bilinear MCR model and the underlying model of the direct multivariate calibration method Classical Least Squares (CLS) are identical and equal to equation (2). The difference lies on the fact that MCR provides the bilinear model $\mathbf{C S}^{\mathbf{T}}$ from the sole information in $\mathbf{D}$ and, in absence of scale information, the concentration and response profiles are in arbitrary units, whereas CLS works with D and reference quantitative information linked to calibration samples, $\mathbf{C}$, to build the calibration model, $\mathbf{S}^{\mathbf{T}}$, that may be used in a subsequent step to predict concentrations in new samples. CLS is the most chemically meaningful calibration method but requires knowing the identity of all components in the samples contributing to the response measured to build correct calibration models. When this is not the case, less interpretable inverse calibration models need to be used. The correlation constraint helps to cover the intermediate situation, i.e., it takes advantage of the reference quantitative information, when available only for some components, keeping at the same time the chemically meaningful MCR bilinear model of the responses measured $[4,32,33]$. The correlation constraint was initially designed to cover first-order calibration scenarios, where every response vector relates to a single sample. In this context, the matrix $\mathbf{D}$ is formed by a set of calibration samples and prediction samples. In every iteration, the correlation constraint is applied individually to every concentration profile linked to a component with reference quantitative information to find the scaling relationship between the concentration values found by the ALS optimization and the reference quantitative information. Once this regression model is found using calibration samples, the real concentration values for unknown samples can be predicted (see Fig. 2b). The correlation constraint has been used for different kinds of spectroscopic [33] and voltammetric data [32] and on examples linked to food samples, artworks, industrial or pharmaceutical applications [33,71-75]. In some of these examples, the correlation constraint has been adapted to deal with sample matrix effects as well $[73,75]$. In all cases, the main aim has not been competing in quantitative performance with inverse calibration methods, such as
PLS [33], but keeping a chemically meaningful model for all components in the data set even if quantitative information is only available for some of them. Another recent approach focused on obtaining concentration profiles in real concentration units for MCR first-order calibration problems make use of soft-known value constraints for the calibration samples that help to obtain concentration values for unknown samples in real concentration units [76].

Recently the correlation constraint has been extended to second-order calibration tasks, i.e., when a full data table relates to a single sample. In this situation, multiset analysis using submatrices of calibration and unknown samples is the normal way to proceed and the quantitative information is extracted from the area under the concentration profiles [17,77]. Again, the areas are in arbitrary units and real concentrations are usually obtained outside the MCR analysis, regressing the ALS areas against reference concentrations. Using the area correlation constraint, the regression model relating the scale of the ALS area to the real reference concentration is performed in each iteration and the concentration profiles are conveniently rescaled to reflect an area equal to the real concentration value [77-80]. When the MCR analysis is finished, the areas under the concentration profiles provide directly the real concentrations of samples. Such a strategy has been proven to decrease the uncertainty in the MCR solutions and, therefore, to improve the quantitation task, especially in multisets formed by non-multilinear data.

Another field of research in the design of constraints is the use of mathematical properties obeyed by the profiles, as it was the case with local rank or selectivity constraints. Recently, sparseness has been tested as an additional constraint clearly linked to a mathematical condition $[50,81,82]$. Usually, sparse versions of chemometric methods tend to use this condition to simplify the calculation load and the interpretation of results, as it happens in sparse exploration or classification methods. However, the use of sparseness as a constraint in MCR methods does not obey this philosophy. Sparseness, understood in this context as the presence of few non-null elements in the recovered profiles, is not a generally applicable condition in MCR analysis and can worsen significantly the fit to the data. However, some response profiles, such as MS spectra, with few $m / z$ significant signals, or concentration profiles linked to distribution maps of minor compounds in hyperspectral images, with presence in few pixels, obey naturally this property [49,50] (see Fig. 2c).

The implementation of the sparseness constraint has been done in different ways, e.g., based on the use of the 0 -norm or the 1norm, and has provided accurate results in contexts where the profiles to be recovered were, by nature, sparse, such as chromatography or imaging based on MS detection. Sometimes, mathematical constraints are also used to favor a certain kind of feasible solution that can be of particular interest for interpretation; this is the case of the angle constraint [83,84]. This constraint forces either the response $\left(\mathbf{S}^{\mathbf{T}}\right)$ or the apportionment profiles $(\mathbf{C})$ to show the maximum angle between them, i.e., to be as dissimilar as possible The direction in which this constraint is applied is related to the kind of information that we need to enhance or understand better, e.g., if the components present in an imaged manuscript are the main goal, contrast may be applied to the $\mathbf{C}$ direction, whereas if MS spectra of different compounds need to be identified, contrast may be applied to the $\mathbf{S}^{\mathbf{T}}$ direction. It is important to realize that the use of the angle constraint does not necessarily provide unique solutions, but it may be useful to explore more clearly the details associated with one of the directions of the bilinear model. Other attempts in the direction to achieve maximum contrast in MCR solutions are, for instance, the use of band target entropy minimization (BTEM) approaches to model the profiles [85]. The 
maximum signal contrast or dissimilarity concepts have also a resemblance with the idea behind Independent Component Analysis based methods [86]. However, independence, like orthogonality, are requirements that are not often fulfilled by the true physical solutions and, therefore, are not suitable for the goals of MCR methods [87].

The emergence of new analytical measurements has also been the seed for the design of constraints based on the natural properties of these new methodologies. A paradigmatic example can be found in the area of hyperspectral image analysis. Hyperspectral images are chemical images of samples that associate a full spectrum with every pixel. As a consequence, they provide chemical and spatial information about images. In their raw form, hyperspectral images are cubes of data, with two spatial dimensions ( $\mathrm{x}$ and y-pixel coordinates) and a spectral direction. Although the data cube is unfolded into a data table $\mathbf{D}$, sized (total nr. pixels, spectral channels), to be analyzed by MCR, the inclusion of spatial information in the resolution process soon appeared to take advantage of this new specificity linked to the imaging measurement. Thus, adapted versions of the local rank constraints using the rank information coming from the local analysis of pixel neighborhood areas in $x$ - and $y$-directions have been designed [51]. And going a step beyond, the modeling of the concentration profiles, refolded into pure distribution maps during the iterative MCR optimization, has allowed the incorporation of spatial or textural characteristics to constrain the refolded pure maps, such as edge-detection, smoothing, or shape constraints when elements in the image obey this characteristic $[49,88,89]$.

\section{New data structures and underlying models}

A crucial milestone in the MCR history was the advent of multiset analysis, i.e., the possibility to use augmented data matrices concatenating different blocks of information. Multiset analysis opened a wealth of possibilities from the chemical point of view, since information from different instrumental techniques, experiments, environmental compartments ... could be interpreted together. Indeed, multiset analysis can be considered an excellent data fusion methodology, born before this term was coined or popularized, and allows taking advantage of the complementary information of the data blocks fused to provide an integral and complete answer to the complex systems analyzed. Multiset analysis was also a major asset to fight against severe limitations related to the analysis of a single data set, such as the very often large presence of ambiguity in MCR solutions and the so-called rankdeficiency problem, associated with linear dependencies among the profiles to be resolved [16,17,19,28,90].

Multiset analysis can be performed on row-wise (equation (3)), column-wise (equation (4)) and row- and column-wise augmented matrices (equation (5)) depending on the direction(s) expanded by the concatenation of blocks of information.

$$
\begin{aligned}
\left(\mathbf{D}_{\mathbf{1}} \mathbf{D}_{\mathbf{2}} \mathbf{D}_{\mathbf{3}} \ldots \mathbf{D}_{\mathbf{L}}\right) & =\mathbf{C}\left(\mathbf{S}_{\mathbf{1}}^{\mathbf{T}} \mathbf{S}_{\mathbf{2}}^{\mathbf{T}} \mathbf{S}_{\mathbf{3}}^{\mathbf{T}} \ldots \mathbf{S}_{\mathbf{L}}^{\mathbf{T}}\right)+\left(\mathbf{E}_{\mathbf{1}} \mathbf{E}_{\mathbf{2}} \mathbf{E}_{\mathbf{3}} \ldots \mathbf{E}_{\mathbf{L}}\right) \\
& =\mathbf{C} \mathbf{S}_{\text {aug }}^{\mathbf{T}}
\end{aligned}
$$

$$
\left(\begin{array}{l}
D_{1} \\
D_{2} \\
D_{3} \\
\ldots \\
D_{K}
\end{array}\right)=\left(\begin{array}{l}
C_{1} \\
C_{2} \\
C_{3} \\
\ldots \\
C_{K}
\end{array}\right) \mathbf{S}^{\mathbf{T}}=\left(\begin{array}{l}
E_{1} \\
E_{2} \\
E_{3} \\
\ldots \\
E_{K}
\end{array}\right)=C_{a u g} S^{T}+E_{a u g}
$$

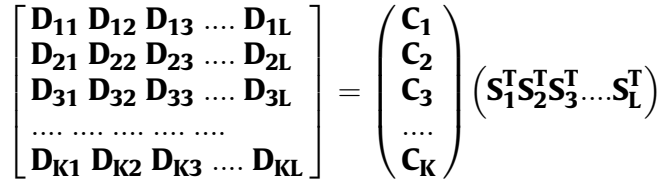

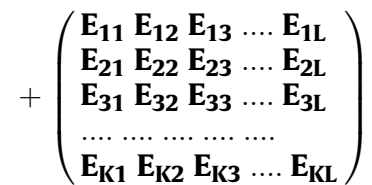

$$
\begin{aligned}
& =\mathbf{C}_{\text {aug }} \mathbf{S}_{\text {aug }}^{\mathbf{T}}{ }^{\mathbf{T}}+\mathbf{E}_{\text {aug }}
\end{aligned}
$$

In these equations, matrices with the subindex aug refer to the concatenated augmented data matrices, those being the original multiset $\mathbf{D}_{\text {aug }}$, or those being the matrices of MCR-ALS resolved concentration profiles or pure responses, $\mathbf{C}_{\text {aug }}$ and $\mathbf{S}_{\text {aug }}^{\mathbf{T}}$, respectively, when they are formed by the concatenation of more than one block of information. Despite the higher complexity of multiset data structures, they can usually be well described by bilinear models and, consequently, MCR is a suitable tool to deal with them. Typical examples of data multisets soon appeared to deal with spectroscopic data fusion associated with process monitoring, where one or several experiments performed in different conditions and monitored simultaneously with several techniques could be combined [90]. In this scenario, the per block flexibility in the application of constraints in MCR-ALS is particularly relevant and allows adapting the algorithm according to the properties of the different spectroscopic techniques, e.g., distinguishing techniques providing positive signals or not [90,91], and to the behavior of the process profiles of the different experiments, e.g., using different hard models and/or soft-modeling constraints [17,29,67,69,90,92]. Another of the first paradigmatic areas of multiset analysis was linked to the solution of second-order calibration problems $[17,77,93]$. Indeed, in second-order measurements, such as hyphenated chromatographic runs or 2D excitation-emission fluorescence landscapes, multisets can be formed by blocks of information related to analyte standards combined with blocks of unknown samples, where one or more analytes need to be quantified. In this situation, the multisets always provide an augmented matrix of concentration profiles and, as mentioned before, the quantitative information is extracted from the area under the concentration profiles, with pseudounivariate calibration lines that are obtained regressing areas from analyte standards against reference concentrations. This procedure allows profiting from the second-order advantage, which enables quantitation of analytes using standards that do not need to contain sample interferences and, in difference to other second-order calibration methods, the MCR solution also provides a characterization of the profiles of the rest of compounds in the samples. MCR for quantitative analysis is also useful in the usual analytical modes, i.e., standard addition method or using internal standards [94]. Along this research line, there has been an evolution in the definition of the figures of merit linked to the quality in concentration prediction. Since quantitative information is obtained from pseudounivariate calibration lines, the first proposals were mere adaptations of quality parameters used in classical univariate calibration [95,96]. Recently, quality in prediction has also been studied using resampling methodologies [97,98]. However, the most remarkable progress has been defining figures of merit that consider the influence of the major or minor overlap of the analyte signal with the interferences in the sample to be predicted. As a consequence, the sensitivity of the method and related parameters, such as limit of detection or quantification, become sample-dependent [99,100]. 


\subsection{Hybrid modeling revisited. Multi-way models for bilinear structures}

The emergence of multiset analysis and multi-way analysis were quite synchronized in time and allowed handling a large amount of related information simultaneously. However, in the beginning, the multiset idea was linked to flexible structures in block size and meaning that were described by bilinear models, whereas multiway analysis needed regular tensors or hypercubes, i.e., with data blocks with more modes in common, that could be analyzed using more complex multilinear or factor interaction (Tucker) models $[17,20,21,24]$. The more complex multilinear models associated with multi-way data analysis had some interesting properties that a pure bilinear model did not offer. For instance, trilinear or its multilinear extension models provided unique decompositions of the original data and a small number of profiles to define all the relevant information of a component, e.g., one for every one of the three modes of the trilinear model [20-23]. Tucker models, although not providing unique solutions, could contemplate the presence of factor interaction, i.e., the fact that two or more components could share a common profile in a particular mode [21,24,25]. Although the choice of an analysis method is driven by the natural behavior of the data and many multisets behave according to a bilinear model, there are instances in which multilinearity and factor interaction are present and Multivariate Curve Resolution has been adapted to these scenarios through the introduction of model constraints [17].

Quite likely, similarly to fluorescence (excitation-emission spectra) for trilinear model based methods in Analytical Chemistry, the analysis of environmental data has been the major trigger for the design and incorporation of model constraints in MCR methods. Indeed, data from environmental monitoring studies can be easily structured as multi-way or multiset structures, where the different modes can be sampling points, physicochemical parameters, time (hours, days, years, ...), .... Each component, i.e., each contamination source in this context, may have a different behavior. For instance, in a multiset formed by blocks of contaminants concentrations collected in different sampling points in different years, the geographical pattern, compositional pattern or time evolution of a contamination source may be defined by a single profile each (trilinear model), or the geographical pattern may change as a function of time (bilinear model), or there may be several contamination sources that share the same geographical or temporal pattern, but differ in composition (factor interaction). This diversity in potential behavior among contamination sources clearly points out that a hybrid modeling tool contemplating all these options is the ideal tool to address environmental data analysis.

Model information encompassing multilinearity and/or factor interaction, has been incorporated in Multivariate Curve Resolution through dedicated constraints. The first constraint implemented was trilinearity and was encoded in such a way that the profiles of the same component in the extended mode of the multiset had to show the same shape [34], e.g., in a multiset formed by excitationemission spectra of different samples where the excitation direction is the augmented mode, all pure excitation spectra of the same compound in the different samples would be forced to show the same shape and would vary only in intensity [101,102]. Recently, a modification of the trilinearity constraint has been implemented allowing for smaller deviations of the perfect trilinear behavior in the profiles constrained [103]. Afterwards, the factor interaction constraint was proposed [36] and there are recent extensions of the trilinearity constraint to obtain quadrilinear models [35,104], and very recently to multilinear models with an unlimited number of linear modes [105]. It is important to note that model constraints are incorporated in MCR on each component separately. This fact allows a per component choice of the model constraint to be used and covers a large range of MCR models that go from a pure bilinear decomposition to hybrid models with components presenting bilinear/multilinear/factor interaction behaviors to complete multilinear models [17]. It is specially in the intermediate hybrid situation, found in analytical and environmental applications, where MCR can be identified as a unique modeling tool, able to address mixed multilinear scenarios that standard bilinear MCR or multiway analysis cannot solve [36,104-107].

Whereas model constraints are oriented to introduce models with more complex structure than the bilinear model, there have also been attempts to progress in the opposite direction proposing solutions based on MCR to treat non-bilinear data sets. These solutions are based on the design of dedicated pretreatments to correct the non-bilinearity of the data or on modifications of the algorithm to adapt to milder deviations of the bilinear condition [108-111].

\subsection{Stretching multiset structures}

Until the present decade, the multiset concept was linked to regular and complete structures that could be well represented by the models in equations (3)-(5). However, when thinking of rowand column-augmented matrices, building a complete multiset is not always possible. The presence of missing blocks of information can be due to inexistent measurements (e.g., not performed in an environmental campaign in a particular period of time), unfeasible measurements (e.g., an instrumental technique cannot perform measurements at the same time scale or, in the same experimental conditions, than another one in a process monitoring framework) or to other circumstances.

Handling multiset data structures with missing blocks of information has become possible by using a variant of the MCR-ALS algorithm adapted to incomplete multiset data structures, as shown in Fig. 3a [47]. The principle behind this variant is that an incomplete multiset can be described as formed by several complete multisets that together cover the blocks of the initial incomplete structure (in the case of the figure, complete multisets $\mathbf{D}_{\mathbf{A}}, \mathbf{D}_{\mathbf{B}}$ and $\mathbf{D}_{\mathbf{C}}$ cover the information of the incomplete multiset $\mathbf{D}$ ). In every iteration, separate factorizations of all complete multisets within the structure are carried out, but a single error function enclosing the sum of squares of the residuals of all factorizations is used to drive the optimization. When some blocks of matrices $\mathbf{C}$ and $\mathbf{S}^{\mathbf{T}}$ are common among factorizations, an average solution is adopted or the solution of a particular factorization is preferred, depending on the kind of problem addressed. In the end, a bilinear model, equal to the one related to a complete multiset, is obtained. This kind of strategy was first applied to an environmental data set, where a temporal monitoring of ozone and nitrogen oxide had inexistent measurements during some years [47]. More recently, it has allowed the combination of chromatographic measurements (with UV and MS detection) and time-dependent spectroscopic monitoring (only possible with UV spectroscopy), for the elucidation of the mechanism and characterization of products in pharmaceutical degradation processes [70]. This approach has also helped to surmount problems related to the image fusion of spectroscopic platforms with different spatial resolution [112].

In a different direction, multiset structures have always been used to combine data matrices. A new problem emerges when matrices and tensors (data cubes) with common modes need to be analyzed together (see Fig. 3b). Up to now, there have been some approaches coming from the multi-way analysis world, such as the Combined Tensor and Matrix Factorization (CTMF), that have tackled this problem $[21,113]$. The way to carry out the analysis is 
a)
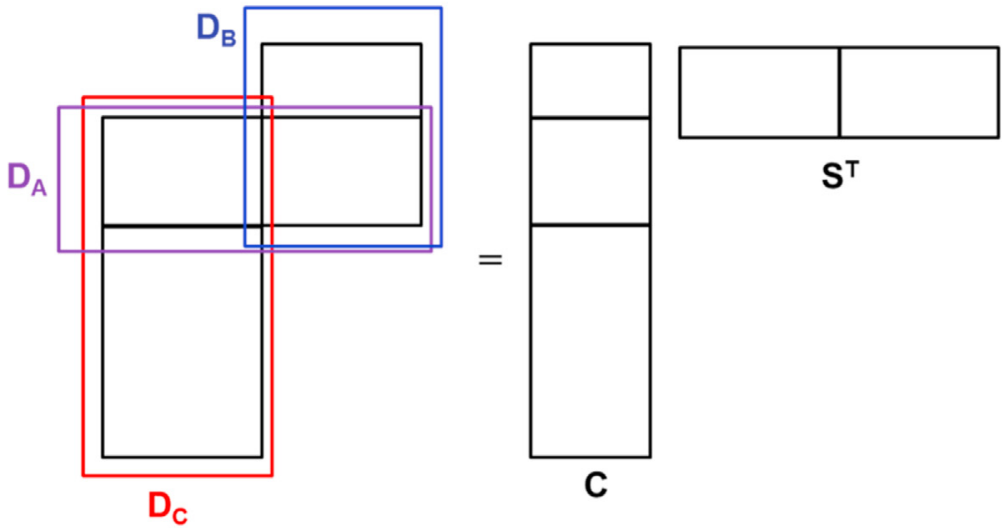

$$
\text { Optimization: } \min \left(s s q\left(\mathrm{D}_{\mathrm{A}}-\mathrm{C}_{\mathrm{A}} \mathrm{S}_{\mathrm{A}}^{\top}\right)+s s q\left(\mathrm{D}_{\mathrm{B}}-\mathrm{C}_{\mathrm{B}} \mathrm{S}_{\mathrm{B}}^{\top}\right)+s s q\left(\mathrm{D}_{\mathrm{C}}-\mathrm{C}_{\mathrm{C}} \mathrm{S}_{\mathrm{C}}^{\top}\right)\right)
$$

b)

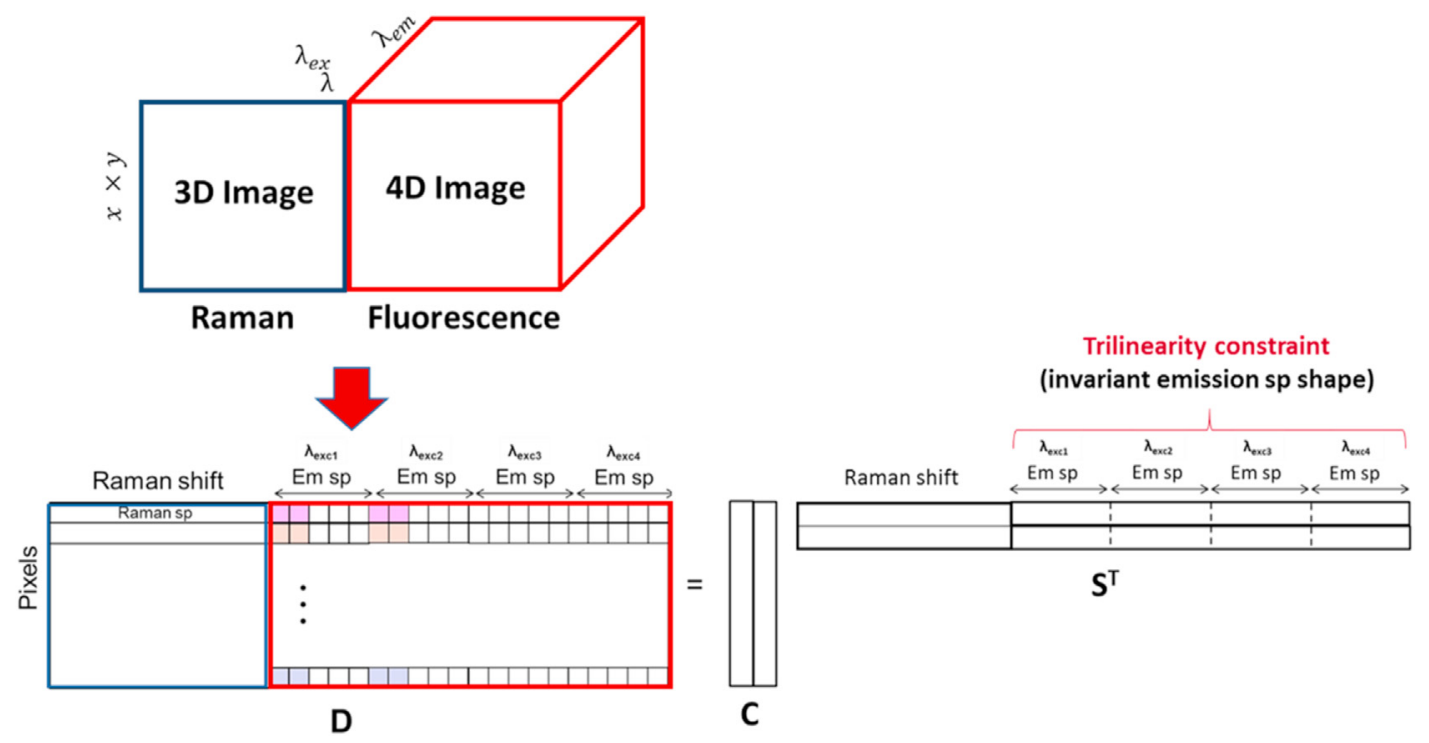

Fig. 3. a) Example of incomplete multiset and MCR optimization (see Ref. [47]). b) Example of fusion of matrix and tensor data sets via tensor unfolding and multiset analysis with hybrid bilinear/trilinear model (see Ref. [48]).

similar in philosophy to the one used in the variant of MCR to deal with incomplete multisets, i.e., separate factorizations of the matrix and tensor are carried out subject to obtain a single set of profiles in the common mode between matrix and tensor and a single error function incorporating the residuals of both factorizations is used. Very recently, MCR has offered a solution to analyze together 2D and 3D data sets that behave according to bilinear and trilinear models, respectively (see Fig. 3b) [48]. It has been applied to connect Raman and 2D EEM spectra in the context of image fusion, where the concentration mode (the pixel mode in images) is common to the two measurements. To do so, a row-wise augmented multiset is built by connecting the Raman spectra and the vectorized 2D EEM spectra of all pixels.

To preserve the natural model of every measurement (bilinear for the Raman data and trilinear for the EEM data) a new modality of hybrid bilinear/trilinear model is used, where the flexibility of the trilinear constraint is seen through an optional per block application. Thus, in the augmented spectral matrix, only the blocks related to EEM measurements, i.e., emission spectra collected at different excitation wavelengths, are forced to obey the trilinearity constraint and present the same shape, whereas the block of Raman spectra is left unconstrained. Working in this way, a single factorization of the multiset with a hybrid bilinear/ trilinear model is enough to solve the problem. Although image fusion has provided the first example of use of MCR to analyze 2D/ $3 \mathrm{D}$ combined data structures, the same strategy may be applied to other applications where bilinear and trilinear measurements are used and also to 3D/3D measurements that share one mode in common. 


\section{Uncertainty in curve resolution. Back to the fundamental problem}

\subsection{Ambiguity in curve resolution. Estimating extent and location}

Since the first work by Lawton and Sylvestre, ambiguity was identified as the Achille's heel of MCR methods, being the main source of uncertainty in MCR solutions [1,2,39,40]. The presence of ambiguity makes that different bilinear decompositions of the same data set with profiles that obey the preselected constraints reproduce the original data set with an optimal fit. These equivalent decompositions provide the range of feasible solutions, all of them equally valid to describe the information in the initial data set. Going in detail, one can distinguish among permutation, scale (or intensity) and rotation ambiguity. The permutation ambiguity refers to the fact that there is no sorting order in the MCR components and, therefore, profiles in $\mathbf{C}$ and $\mathbf{S}^{\mathbf{T}}$ matrices can be shuffled (always keeping the right correspondence of the dyads $\mathbf{c}_{\mathbf{i}} \mathbf{s}_{\mathbf{i}}^{\mathbf{T}}$ ) and provide identical results. The scale ambiguity is well represented in equation (6) and describes that dyads of profiles having the same shape but different relative scales between concentration profile and pure response profile ( $k_{i}$ scaling factor) reproduce equally well the original data set. Finally, the rotation ambiguity, expressed in equation 7, describes that bilinear models of $\mathbf{C}$ and $\boldsymbol{S}^{\mathbf{T}}$ matrices with profiles differing in shape and obeying the preselected constraints can present the same model fit. In equation $7, \mathbf{R}$ is a transformation matrix that does not modify the reproduction of the data set but modifies the shape of the $\mathbf{C}$ and $\mathbf{S}^{\mathbf{T}}$ profiles [39,40,52].

$$
\mathbf{D}=\sum_{\mathbf{i}} \mathbf{c}_{\mathbf{i}} \mathbf{s}_{\mathbf{i}}^{\mathbf{T}}+\mathbf{E}=\sum_{i}\left(\boldsymbol{c}_{\boldsymbol{i}} k_{i}\right)\left(\mathbf{s}_{\mathbf{i}}^{\mathbf{T}} \frac{1}{k_{i}}\right)+\mathbf{E}
$$

$$
\mathbf{D}=\mathbf{C S}^{\mathbf{T}}+\mathbf{E}=(\mathbf{C R})\left(\mathbf{R}^{-\mathbf{1}} \mathbf{S}^{\mathbf{T}}\right)+\mathbf{E}
$$

Ambiguity is generally decreased or suppressed by the action of constraints $[19,39,40]$. In the case of scale ambiguity, the problem is easily solved by normalizing the profiles in one of the modes or applying closure (when suitable). Rotation ambiguity has always been the major and most difficult to solve source of ambiguity. Along the history of MCR, there has been research oriented to know which constraints or data set structures can ensure solution uniqueness [1,2,39,40,52]. A very relevant contribution was done by Manne in the 90's with his resolution theorems that described the necessary rank overlap conditions among the profiles of components to retrieve correct and unique profiles [18]. Such a contribution confirmed the essential role of local rank and selectivity constraints to decrease uncertainty in MCR solutions [19]. Although Manne's theorems were thought for the analysis of a single data matrix, this knowledge has served afterwards to help in the design of multiset structures that could provide the sought rank overlap conditions by adding standards or suitable information [114,115]. Manne's theorems and the relationship between concentration and response spaces have been more recently revisited through the socalled duality concept that helps to understand the theoretical basis of the influence of the compound overlap pattern in one space over the uncertainty on the profiles retrieved in the counterpart space of the bilinear model $[116,117]$. In addition to local rank and selectivity conditions, other constraints, such as trilinearity or hard modeling, are known to provide unique solutions in MCR problems [39]. Another known fact is that the use of multiset analysis decreases ambiguity when compared with analysis of single matrices [19,118]. However, there are many other scenarios where ambiguity exists, and it is important to have tools that can help to investigate this phenomenon.
There is nothing more ambiguous than saying that MCR provides ambiguous solutions. Other than a pun, this statement reflects that a proper definition of ambiguity needs to be provided per component and per profile in the bilinear model. And it is not only a question of location, but the extent of ambiguity is also important to assess the uncertainty of the profile recovery. The last two decades have devoted an intense effort to design tools for the proper description and assessment of the ambiguity phenomenon. As mentioned in the history section, there are two tendencies in this research field: a) methodologies oriented to find extreme values (maximum and minimum) in the MCR profiles obtained [37,38] and b) methodologies devoted to find the full range of feasible solutions [39,40,52]. Whereas the former option is more practical and closer to the concept of analytical uncertainty linked to other data analysis methods, the real truth is that the sole dyad of a maximum and a minimum profile cannot properly display all the variability in profile shape of the range of feasible solutions of every component because usually more than these two extreme profiles are needed to describe them graphically or geometrically. Only in the case of two component systems, these extreme profiles will enclose all the set of feasible solutions. Therefore, both approaches have pros and cons and can be considered complementary.

The approaches oriented to find minimum and maximum feasible profiles are based on finding, per each component, the dyad of concentration profile and pure response that belongs to a feasible MCR solution obeying the preselected constraints and provides the minimum or the maximum of an objective function [37,38]. Often, the objective function is related to the signal contribution of the component to be optimized, expressed as the 1-norm of the outer product of the dyad $\mathbf{c}_{\mathbf{i}} \mathbf{s}_{\mathbf{i}}^{\mathbf{T}}$ in Gemperline's approach [37], or with the ratio of the 2-norm of the outer product of the dyad of the component over the 2-norm of the original data set $\mathbf{D}$ in the socalled signal contribution function (SCF) in Tauler's approach [38]. These methodologies clearly answer whether the dyad of profiles of each component is recovered in a unique way (when there is no difference between the maximum and minimum signal contribution) or not (when this difference is higher than zero). The difference between maximum and minimum signal contribution is also a quantitative indicator of the extent of ambiguity for a component. Besides, if the concentration or the response profile of a component is uniquely recovered, this can be seen because the profiles linked to the maximum and minimum signal contribution are identical. Gemperline [37] and Tauler [38] have been the main promotors of this kind of approach and there are now freely available interfaces that help to estimate ambiguity in the MCR solutions in this way $[119,120]$. The main advantage of this approach is that there is no limit in the number of components of the system studied. Besides, the optimization used to find the extreme profiles allows for the implementation of a large variety of constraints for a very complete test of ambiguity in a wide variety of resolution conditions [121]. Observe that in this work the term 'boundaries' has been avoided for the reasons explained above about the impossibility to define the whole set of solutions by two extreme profiles defined in terms of a maximum and minimum of an objective function. Different attempts to understand the position of these extreme values in the geometrical representations of the full range of feasible solutions (see below) have been presented [122].

Many of the approaches devoted to find the full range of feasible solutions derive directly from the seminal work of Lawton and Sylvestre, related to the study of a two-component system [1], and the follow-up by Borgen and Kowalski, who extended the concept to three-component systems [2]. In these cases, the area of feasible solutions (AFS) for concentration and response profiles were found in the scores and loading spaces, respectively, subject to nonnegativity and normalization of profiles in one of the modes. For 
two- and three-component systems and under the constraints mentioned, there is an analytical solution to obtain the boundaries of AFS. Although these analytical solutions and the related conceptual geometric theory were revised some years ago [123], new algorithms have been proposed to find numerically the external boundaries of the area of feasible solutions, also in instances where additional constraints enter into play. All these methodologies use the relationship of concentration and response profiles with the scores (US) and loadings (V) space, expressed as, $\mathbf{C S}^{\mathbf{T}}=\mathbf{U S V}^{\mathbf{T}}$ where the left-hand side of the expression is the MCR bilinear model and the right-hand side is the Singular Value decomposition model, where $\mathbf{U}$ is the matrix of normalized scores, $\mathbf{S}$ the matrix of singular values and $\mathbf{V}$ the loading matrix. Using a transformation matrix $\mathbf{T}$, we can express that $\mathbf{C}=\mathbf{U S T}$ and $\mathbf{S}^{\mathbf{T}}=\mathbf{T}^{-\mathbf{1}} \mathbf{V}^{\mathbf{T}}$, i.e, every feasible solution has a related $\mathbf{T}$ matrix. In all the approaches proposed, the optimization steps to find AFS boundaries are performed working with the small squared matrix $\mathbf{T}$, sized ( $n c \mathrm{x} n c$ ), where $n c$ means number of components of the MCR model. Basically, all these methodologies start with the $\mathbf{T}$ matrix related to the solution obtained by the MCR analysis or to any other feasible solution, and via different kinds of optimization find the $\mathbf{T}$ matrices that allow defining the area of feasible solutions. The size of this area and the shapes of $\mathbf{C}$ and $\mathbf{S}^{\mathbf{T}}$ profiles contained in it give a good idea of the extent of ambiguity and the variability of the feasible profile shapes, respectively. There has been a significant improvement of the algorithms used for this task in order to improve the computation time and accuracy in the determination of the AFS border. The first algorithm, proposed for a two-component system, was based on an exhaustive grid search of the full area of feasible solutions [124]. To tackle systems with a higher number of components, less computationally intensive methods were proposed that focused only on describing accurately the AFS border, knowing that points inside these limits do not provide much additional information. The first attempt used a simplex-based algorithm, starting from the MCR solution to reach a point in the border of AFS and then used an adapted simplex algorithm to complete the AFS perimeter $[125,126]$. A related improvement to this algorithm was provided by the polygon inflation method that approximates the border of the AFS by a sequence of growing polygons from the initial MCR solution and a new alternative based on the use of a ray casting method that can, in principle, be theoretically implemented for systems with any number of components [127,128]. However, it has to be mentioned that, in practice, these approaches have been applied only to the analysis of three- and four-component systems. In addition to the mathematical complexity to perform these calculations and the extreme difficulty to find ways to visualize the results for systems with a higher number of components, the AFS for these complex systems is often defined, as seen in examples of 4component systems [128], as a continuous topological entity instead of component-specific separate AFS regions, which hinders a clear interpretation of the ambiguity phenomenon. Up to now, the intensive and complex work developed along this research line has resulted also in a free available interface that helps in these calculations to the non-experienced users [129]. A throughout comparison among the different methods is given in Ref. [40].

The methodologies and practical tools developed to estimate ambiguity by the different methodologies mentioned above have helped to know much more about the effect of many of the constraints developed for MCR methods in the uncertainty of the final solutions. Other than the study of classical constraints, such as nonnegativity, unimodality, local rank, closure or equality constraints $[19,39,40,52,130,131]$, there are recent works that clearly describe the effect of using hard-modeling information [132] and the influence of image-specific constraints in the ambiguity of MCR solutions [133]. It is also interesting to mention the research devoted to describe the effect of rotational ambiguity in quantitative analysis by MCR [134,135]. In the referenced works, estimates of the error in the concentrations of the different components in secondorder calibration based on MCR are found via a dedicated modification of the objective function to be maximized or minimized to estimate the rotational ambiguity. The new function in this context is defined as the ratio of the component area under its concentration profile over the sum of all component areas.

Even in the absence of ambiguity, as in any other analytical method, there exists an uncertainty in MCR solutions linked purely to the propagation of the experimental error of the initial measurement. This contribution can be estimated using resampling methodologies, such as bootstrap or Monte Carlo-based approaches [97]. One has to be aware that, in the presence of ambiguity, the solutions provided by resampling methodologies mix the uncertainty purely due to error propagation with the ambiguity in the solutions obtained [136]. In absence of rotation ambiguities, the estimation of the uncertainties in the MCR model parameters (C and $\mathbf{S}^{\mathbf{T}}$ ) due only to propagation of experimental error can be obtained by these resampling methods. There are interesting resampling studies that help to note how the increase of noise level in the original measurement can lead to the emergence of ambiguity when there was none or to a substantial increase of this phenomenon in the sought solutions [137]. Current efforts are pursued to clear out the complex mixed effects produced on MCR solution uncertainties by the simultaneous presence of rotational ambiguity and experimental noise and to have methods for their evaluation.

\subsection{Taking noise into account. Weighted modeling}

Noise level and noise structure play a significant role in the results obtained by any data analysis methodology. In the initial design of many of the MCR algorithms based on ordinary least squares principles, the inherent assumption was that the data sets to be analyzed were affected only by the presence of i.i.d.-noise. In this scenario, weighting schemes were not necessary. This assumption, which is taken for granted in many of the currently published works, does not pose serious problems when the noise level of the measurements is not very high. When this is not the case, e.g., in some environmental problems or in the analysis of instrumental measurements with a low signal-to-noise ratio, the noise structure really matters and can easily lead to biased models, overfitting and to the significant modification of the shape of the profiles retrieved by MCR.

A first step in using weighting approaches for MCR tasks is knowing the real level and structure of noise in the data sets. Indeed, the use of weighting approaches with bad estimates of noise is more harmful than the mere use of unweighted approaches. Connected with this problem, Wentzell has provided a complete and clear conceptual framework to classify and detect noise structure in data sets [138]. In his formulation, it is noted that noise structure can vary among concentration and response directions or even at individual row or column level in the original data set. Besides, the different noise structure (independent or correlated) has consequences in the structure and use of error covariance matrices in the calculations used for weighting approaches.

Some of the weighted variants of MCR algorithms are based on least-squares approaches, such as Multivariate Curve ResolutionWeighted Alternating Least Squares (MCR-WALS) [139,140]. In these cases, when $\mathbf{C}$ and $\mathbf{S}^{\mathbf{T}}$ matrices are calculated, the suitable error covariance matrix is incorporated in each least-squares calculation. Since these operations can be computationally intensive and lengthen significantly the use of iterative MCR approaches, it has been proven that the classical weighted variant of MCR can be 
replaced by a strategy that works starting with an initial Maximum Likelihood PCA (MLPCA) weighted filtering on the original data set to continue with the classical MCR-ALS optimization [141]. There exist also other weighted MCR algorithms that are not based on least-squares calculations, but on other kinds of optimization like the Newton-Gauss algorithm for different variants of Positive Matrix Factorization (PMF) [142], a modified conjugate gradient algorithm for the multilinear engine method [143], or the log likelihood maximization for MCR-log likelihood maximization (MCR-LLM) [144]. More recently, other statistically based approaches not based on weighted least squares but on Bayesian methods have been also proposed to solve the source apportionment factor analysis (mixture analysis) problem and provide simultaneously reliable estimations of the uncertainties in the model parameters (C and $\mathbf{S}^{\mathbf{T}}$ ) [145].

Most of the applications of weighted variants of MCR are associated with receptor modeling problems in environmental studies, i.e., with the modeling of profiles aiming at the definition of contamination sources $[140,142,145]$. In these cases, many of the environmental parameters measured have different uncertainties and, often, concentrations of minor contaminants show a rather low signal-to-noise ratio, a scenario that clearly demands the use of weighting schemes. Other common examples where the use of weighted MCR variants have been found justified is the interpretation of genomic data $[139,146]$ where outputs of DNA microarrays show a high level of non-iid noise, or certain kinds of spectroscopic measurements that show low-signal-to-noise ratio and specific kinds of source-related noise, such as ultrafast spectroscopies [147].

\section{New curve resolution domains. Current analytical challenges and big data}

Making a simple search in Web of Science using the term Multivariate Curve Resolution and similar related variants, we can find more than 3200 works, 1000 of them in the last five years in different application domains that range from chemistry and spectroscopy (the most common ones), to biology [54,64,91,139,146] environmental sciences $[28,101,106,140,142]$ or pure mathematical or fundamental theory $[1,2,37-40,79,114,117,118,121-138]$ touching also many applied fields such as pharmaceutical studies or food chemistry $[33,43,54,73]$ and areas that may be as unexpected as cultural heritage $[71,148]$. Such a result confirms the vocation and potential of the MCR methodology to be a versatile tool that can be adapted to the natural characteristics of a large diversity of problems.

In many applications, the recovery of response and concentration profiles, whichever their problem-related meaning, is the ultimate response. However, there is an increasing use of $\mathbf{C}$ and $\mathbf{S}^{\mathbf{T}}$ profiles as seeding information for many other data analysis purposes. Indeed, resolved MCR profiles (scores, $\mathbf{C}$, and loadings, $\mathbf{S}^{\mathbf{T}}$ ) are excellent compressed information that represent adequately the initial information of the data set and, in difference with analogous representations coming from PCA or ICA, the information enclosed in the MCR profiles is compound-specific and chemically meaningful, two relevant properties for many applications.

Until 2000, the traditional niches of application of MCR were process analysis problems, where the word process needs to be interpreted as any evolving chemical system, like a chemical reaction, physical transformation or a chromatographic elution can be, and environmental data sets. In the $\mathrm{XXI}^{\mathrm{st}}$ century, the progress in these application domains continues, but new areas of application linked to complex analytical measurements, such as multidimensional chromatography or hyperspectral image analysis, or to the interpretation of massive data sets linked to -omics studies in systems biology have strongly burst into the MCR arena. Nowadays, it is impossible to describe exhaustively the wide catalog of MCR applications and the next paragraphs only aim at pointing out some relevant advances that can illustrate the current power of this methodology.

In the process monitoring world, data fusion involving different kinds of experiments and/or multisensor monitoring is continuously increasing and focuses on understanding complex processes, such as those present in biochemical or environmental systems or linked to industrial monitoring and control [91,92,149-158]. Sometimes the complexity is intrinsic to the measurement and non-linearities or serious artifacts need to be compensated, like it happens in NMR [109], some electrochemical techniques [108] or in ultrafast spectroscopies [67,110]; other times it is linked to the process evolution itself, which can be difficult to model with a classical hard-modeling framework but may allow deviations from it, like in biochemical [86,152-158] or industrial systems [72,149]. Research also leads to explore the combination of less common kinds of experiments, such as chromatography and sensor monitoring $[69,152]$, or to handle the absence of blocks of information [70].

Within the chromatographic context, the size and complexity of the chromatographic runs has significantly increased. The higher resolution in MS detection systems has allowed the possibility to resolve runs with hundreds of compounds for purposes of qualitative and quantitative characterization of complex samples, in the context of targeted and untargeted analyses. To do that, variants of MCR that incorporate strategies of data filtering and compression, such as the search of the Regions of Interest, ROI, have been used [159]. The combination of MCR and ROI approaches in the recently proposed ROIMCR method [160], has opened the possibilities of simultaneous targeted and untargeted analysis of huge MS data sources (from MS chromatography and MS imaging), in omics studies (metabolomics, lipidomics, proteomics), and also in environmental studies (environomics) where hundreds of sample constituents can be simultaneously characterized [161].

There is an increase in the variety and combination of detection systems in separation techniques that leads to new data structures and analysis, such as the four-way data linked to analysis of several chromatographic runs with excitation-emission detection $[162,163]$, or to multiset analysis that connects different detections in tandem $[68,150,164]$. Chromatography also sees a relevant preprocessing area linked to run alignment by warping methodologies when methods with multilinear models need to be used $[165,166]$. The use of MCR scores in this context is closely related to secondorder calibration tasks, as previously mentioned $[17,77,93,167]$. It is also in this quantitative context that the area correlation constraint has found most applicability [17,77-80].

In environmental analysis, MCR falls into the ensemble of tools for receptor modeling [140,142,168]. In this field, hybrid modeling involving multilinear or factor interaction models [35,36,101] use of noise-related weighting approaches [140,142,143] and analysis of incomplete multisets [47] were already mentioned. Algorithms such as Positive Matrix Factorisation [27] are widely used and Bayesian approaches to incorporate spatial correlation in multisite multipollution sources are also proposed [145]. Bayesian methods offer a great potential because they allow estimating simultaneously the unknown number of factors, and the parameter uncertainties due to rotational ambiguity and noise propagation [169]. There are also other approaches that combine receptor modeling with deterministic contaminant transport models within the algorithms [142]. The MCR-derived geographical profiles linked to pollution sources are another example of use of MCR scores as seeding information for Geographical Information Systems (GIS) [170] or as starting information for deterministic environmental modeling approaches [171]. MCR has contributed significantly in the retrieval of chemically meaningful source apportionment 
a)

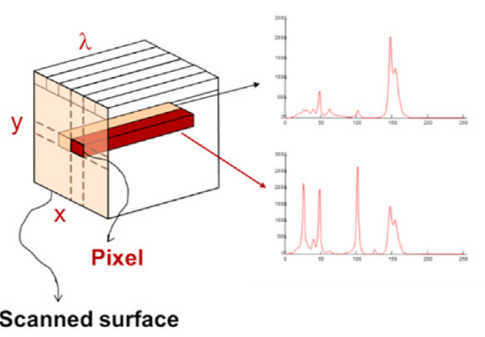

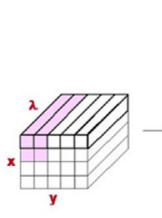
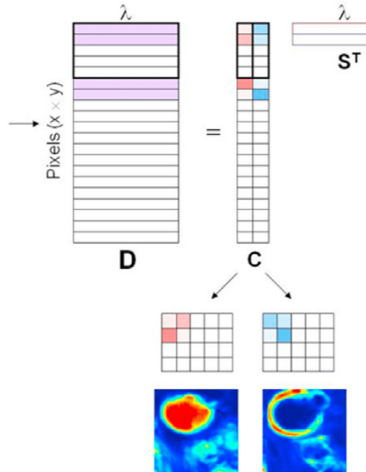

b)

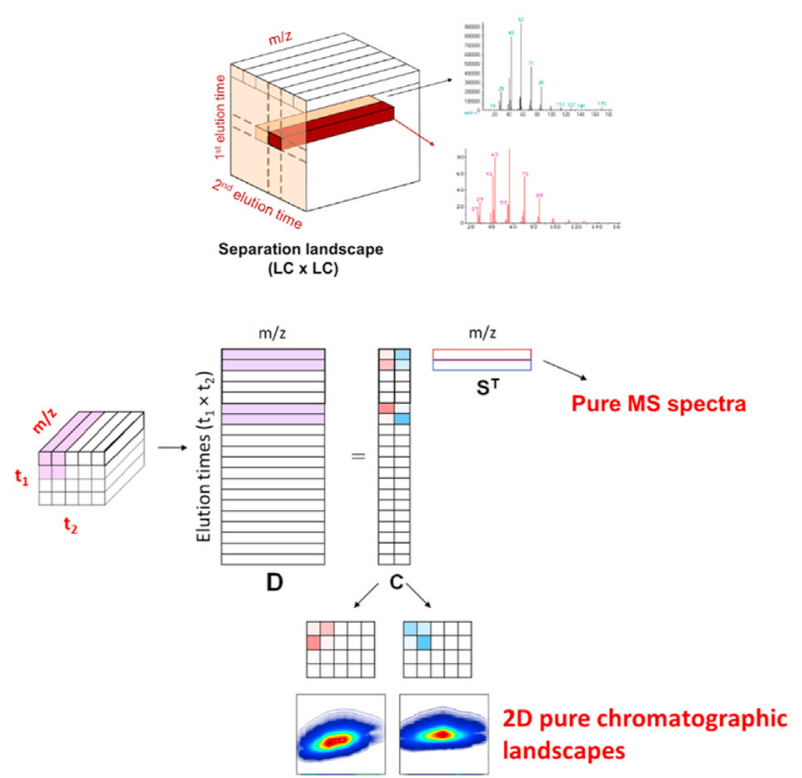

Fig. 4. MCR models in a) Hyperspectral image analysis and b) two-dimensional chromatography.

models in data sets showing a large variety of modes (sample sites, seasons, environmental compartments, ...) through the large variety of data set structures and models described.

Recent applications of MCR are devoted to the analysis of complex analytical measurements, such as hyperspectral images [172] and multidimensional chromatography [44-46,173]. These two types of measurements provide initially 3D structures: in the case of images two dimensions are related to the pixel coordinates and the third to the spectral direction, whereas multidimensional chromatography has two elution directions and a third detection direction (typically MS or DAD). MCR tackles these two structures by unfolding the initial cube into a data table where the nonspectroscopic dimensions are unfolded (see Fig. 4).
The large amount of spectra is decomposed by MCR and the concentration profiles in the bilinear model can be subsequently refolded to recover the $2 \mathrm{D}$ distribution maps in images and the $2 \mathrm{D}$ pure elution landscapes in multidimensional chromatography. Other than this shared characteristic, each one of these fields has promoted the emergence of specific advances.

In the case of hyperspectral image analysis, it is worth mentioning that unmixing methodologies coming from the remote sensing community are developed to perform the same task as MCR does [174,175]. Within the MCR algorithms, the spatial dimension of the measurement has been actively used through the generation of a wealth of spatial constraints [49-51,88,89,176]. Image fusion has allowed working with images recorded with the same platform in order to perform process monitoring [177,178], sample multilayer analysis [179] or quantitative determinations of compounds at global image and local pixel levels, to mention a few examples $[77,172,180]$. More challenging has revealed the image fusion of different spectroscopic platforms that requires a common pixel mode $[181,182]$. In this context, suitable preprocessing to achieve this fact (by performing spatial transformations and binning) is required [183-185]. In some cases, the pixel downsampling step to balance spatial resolutions among the platforms in the multiset analyzed by MCR is followed by a least-squares step using the resolved signatures and the pixel spectra of techniques with higher resolution to recover the $2 \mathrm{D}$ maps with the original spatial resolution of each platform [185]. Other approaches propose image fusion strategies that handle differences in spatial resolution by means of a single incomplete multiset analysis [112]. Image fusion of images with 1D and 2D spectral information has also been approached by using hybrid bilinear/trilinear models, as shown in Fig. 3c [48]. Another advance has come from the inclusion of information of greyscale or RGB images in the MCR analysis to complement the spectroscopic measurements. This option has been used by implementing constraints based on this kind of images [186] or by appending them to the multiset structure [185]. In the image analysis field, the use of MCR scores, i.e., pure distribution maps, has been the seeding information for image segmentation approaches [187], quantitative analysis and superresolution strategies [180,188]. Finally, hyperspectral images have large size and there is a significant effort devoted to work with smaller representative fractions of the image measurement to speed up the MCR analysis. The use of the ROIMCR approach is suitable for MS images $[189,190]$. Other approaches aim at selecting essential pixel information to do a fast MCR analysis that provides the pure spectral signatures in $\mathbf{S}^{\mathbf{T}}$, which are subsequently used to retrieve the distribution maps by a single least-squares step using the information on the full initial image. This search of essential pixels is sometimes based on convexity concepts or on adaptations of purest variable search [191,192].

In the case of multidimensional chromatography, there are two main approaches depending on the data structure and models used: those that leave the multidimensional array in the original form and try to apply multilinear models, such as PARAFAC or direct trilinear decomposition methods [45,193,194], and those that unfold the original chromatographic structure leaving an extended mode for one of the chromatographic dimensions and work using a bilinear model [46,195-197]. The first kind of methods is often applied to GC $\times$ GC-MS, where data are very close to the trilinearity assumption in many examples; when this is not the case or in LC $x$ LC data, the use of trilinear methods needs a dedicated preprocessing oriented to correct peak misalignments, such as $2 \mathrm{D}$ warping methodologies or similar methods [198,199]. The unfolding followed by MCR bilinear decomposition does not require any pretreatment and respects the natural behavior of the chromatographic elution, allowing for peak shifts and shape changes if 

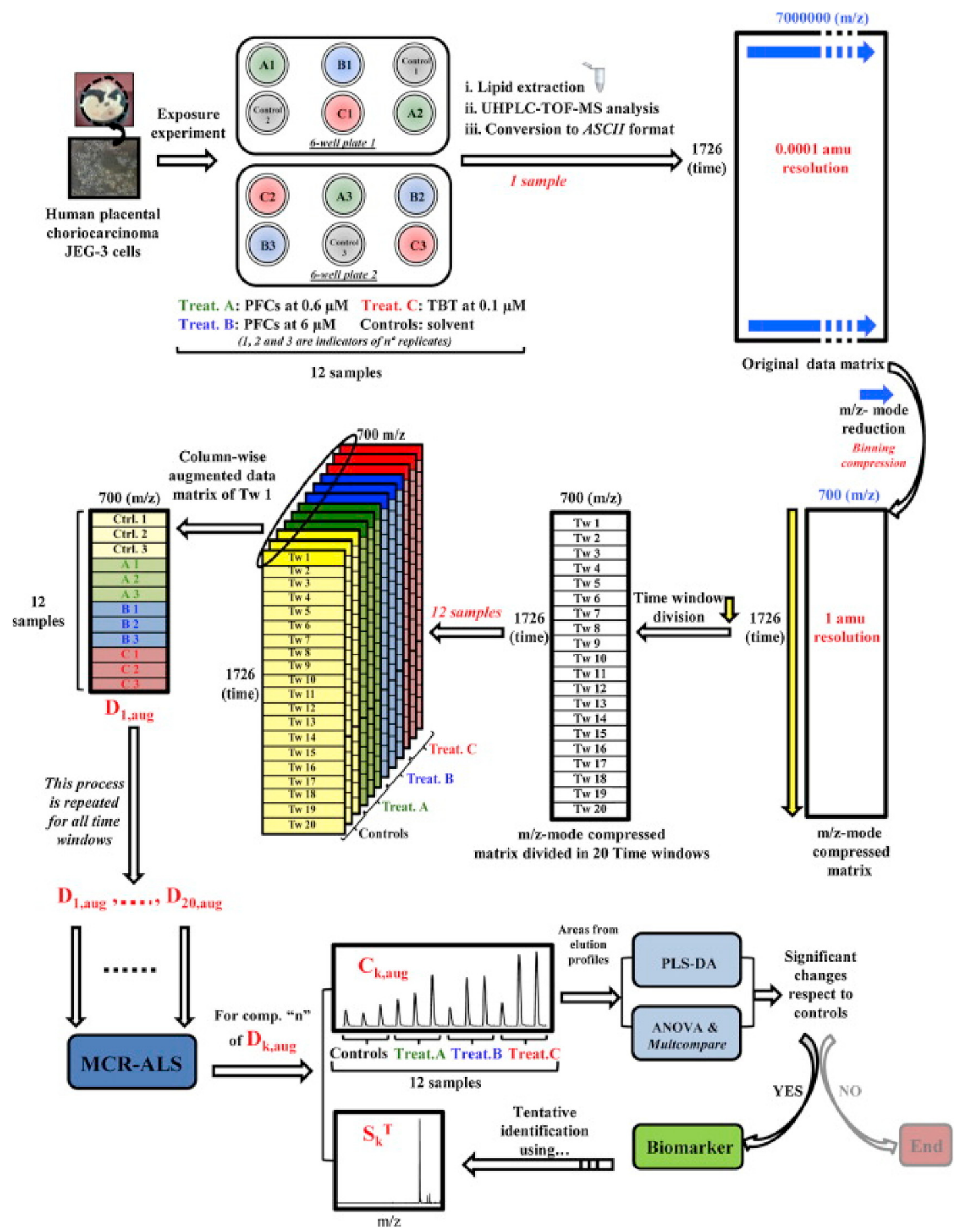

Fig. 5. -Omics data analysis pipeline involving MCR to characterize metabolites $\left(\mathbf{S}^{\mathbf{T}}\right)$ and use MCR quantitative scores for PLS-DA or ASCA models (reprinted from E. Gorrochategui, J. Casas, C. Porte, S. Lacorte, R. Tauler, Anal. Chim. Acta 2015, 854, 20-33.).

existing. The most recent tendencies seem to go in the direction of the bilinear modeling because there is a clear complexity in the alignment step, particularly for systems with a high number of overlapped components and, by nature, many multidimensional chromatographic examples do not obey the trilinear condition, since not only peak shift, but also peak shape changes may exist in the unfolded chromatographic dimension. Another concern in dealing with multidimensional chromatography is the data size. In this respect, compression in the MS and/or in the elution direction is carried out before resolution takes place. In recent times, there are also many studies devoted to see how to use MCR results for third-order calibration purposes [162,200]. Indeed, in the multiset context, one has to be aware that when several samples are analyzed, multidimensional chromatography provides a four-way array, i.e., the information linked to every sample is formed by a 2D elution landscape or by several blocks of unfolded elution profiles. The use of MCR approaches for multidimensional chromatography has been applied to the different modalities of this 
analytical technique $(\mathrm{GC} \times \mathrm{GC}, \mathrm{LC} \times \mathrm{LC})$, with different detection (MS, UV ...) and recent works also show works with UV and MS detections in tandem [173].

MCR has played an essential role in the interpretation of -omics data sets during the last decade. This application domain provides massive data sets that incorporate the results of multiple experiments specifically designed to test the effect of one or more factors in one or more sample populations formed by organisms in the same or different clinical/environmental/conditions. Every experiment in the designed study provides samples, e.g., pooled organism extracts, biological fluids, tissues ... that are subsequently analyzed by different analytical platforms in order to know the desired -oma of the samples. Genomic studies imply the use of DNA microarrays and due to the low signal-to-noise ratio and the Poisson noise associated with this analytical measurement, weighted MCR variants need to be applied to ensure reliable results. MCR provides basic gene profiles related to apportionment profiles linked to a different extent to DNA sequences coming from particular cell lines. In this case, the MCR gene profiles are subsequently used to detect the over- or infraexpressed genes associated with the cell lines more prevalent in the related apportionment profile [139,146,201,202]. In metabolomic studies, separation techniques in all its diversity (GC [54,203], LC [53,160,204,205] capillary electrophoresis [206,207] or multidimensional chromatography $[44,195,208,209])$ with MS detection are the preferred platforms, although the use of NMR is also found in a good number of works. -Omic studies monitored with separation platforms need to follow a complex data analysis pipeline to provide reliable results (see Fig. 5). First, it is needed the suitable compression of MS information in the multiple chromatographic runs. Subsequently, an MCR multiset analysis of all runs collected in the metabonomic study is carried out. Due to the high resolution of the MS detection, MCR analyses can provide as a result a good resolution of a large number of components (hundreds), which helps to obtain a good metabolomic profiling of the different kinds of samples analyzed.

This strategy can be done in targeted or untargeted analysis modes [53], or even in both simultaneously. In this context, MCR on -omics separation data provides pure MS signatures that allow for identification of metabolites (especially useful in untargeted approaches). Besides, when data have been adequately scaled and normalized, the areas under the elution profiles of the metabolites provide compositional data that can be used to test whether there are statistical differences among the metabolome of the samples in the different conditions tested.

The compositional information, i.e., the metabolite areas directly extracted from the MCR elution profiles, can be compared in a classical one-at-a-time individual metabolite test. However, it is more interesting to use all compositional information together as a seeding information for multivariate analysis methodologies such as PLS-DA, e.g., to detect metabolite biomarkers that may explain differences among control and stressed samples, or in more complex data analysis approaches, such as those based on ANOVASimultaneous component analysis (ASCA) when the effect of more than one factor at different levels wants to be assessed [195,210,211], in ANOVA-TP [212] or in general using Generalized Linear Models [213]. A similar strategy is used when NMR data are analyzed. In this case, an NMR spectrum is associated with each sample and MCR provides NMR profiles of pure metabolites directly associated with concentration values in the different samples that may be subsequently compared to assess the effect of the factors or experimental conditions in the metabolome [214-216]. Hyperspectral image analysis has also been used to assess how the fingerprint of tissues was changing by metabolic stressor agents [217,218].

Examples of metabolomics studies and use of MCR can be found in clinical and environmental contexts [219]. It is important to note that, in metabolomics, MCR can be considered almost as a preprocessing step, aiming at providing unmixed reliable qualitative and quantitative information on individual metabolites. The pure response signatures are confronted with databases for metabolite identification and the related quantitative information is used to statistically detect metabolic biomarkers that, once found, can give hints related to the metabolic pathways affected by the particular factors or stressing agents under study.

\section{User-friendly MCR implementations}

An essential reason for the widespread and increasing use of the MCR methodology is that the progress in the theory and application fields of the algorithm has been accompanied by the design of many user-friendly implementations of the method that can be found in different contexts and formats. Indeed, MCR can be easily found (with more or less flexible implementations) in the toolbox of most specialized commercial chemometric packages and even in some instrumentation software. However, it should be taken into account that there are several Graphical User Interfaces (GUIs) available for free that often incorporate the latest advances of the algorithm in the subsequently updated versions. Other than the two versions of MCR-ALS that are specifically linked to the implementation of this algorithm [120,220], other toolboxes devoted to more generalized chemometric software, such as packages for second-order multivariate calibration methods include the algorithm [221,222]. Many of these interfaces are stand-alone GUIs programmed using the MATLAB platform, although other softwares encoding the algorithm in R [205] or Python languages can be found [223]. MCR also appears encoded in softwares devoted to very specific applied areas, such as chromatographic data analysis [224] or hyperspectral image analysis [225,226]. Besides, uncertainty in curve resolution is already encoded in available GUIs $[119,120]$ and specific packages developed for this purpose [129].

\section{Conclusions}

The advance of MCR during these 50 years is due to the joint effort of many researchers coming from different domains and scientific backgrounds that have contributed to design a versatile and ever-growing methodology. The years to come will surely see developments in theoretical concepts and domains of application. Challenges like the adaptation to big data will play a dominant role and we might see that the border among multiset, multi-way and matrix and tensor factorizations gets thinner and perhaps a generalized modeling framework is achieved. The interdisciplinary dialog on the mixture analysis problem may also grow and a fruitful exchange of modeling perspectives between the MCR community and the image unmixing remote sensing, environmental receptor modeling or blind source separation in signal processing can be of use to all agents involved. Best ways to assess rotation ambiguities and parameter uncertainties in MCR solutions will be encountered and accepted by the chemometrics community. Quoting Charles Mingus, an American bass player: "Making the simple complicated is commonplace; making the complicated simple, awesomely simple, that's creativity". MCR is probably the simplest method to address the mixture analysis problem: let's keep creating!!

\section{Declaration of competing interest}

The authors declare that they have no known competing financial interests or personal relationships that could have appeared to influence the work reported in this paper. 


\section{Acknowledgements}

Authors acknowledge the Ministerio de Ciencia e Innovación (MINECO) from the Spanish government for financial support through the projects PID 2019-1071586B-IOO and PID 2019105732 GB-C21.

\section{References}

[1] W.H. Lawton, E.A. Sylvestre, Self modeling curve resolution, Technometrics 13 (1971) 617-633.

[2] O.S. Borgen, B.R. Kowalski, An extension of the multivariate componentresolution method to three components, Anal. Chim. Acta 174 (1985) 1-26.

[3] Z. Zhang, P. Ma, H. Lu, Two-way data analysis: multivariate curve resolution: noniterative resolution methods, in: S. Brown, R. Tauler, R. Walczak (Eds.), Comprehensive Chemometrics vol. 2, Elsevier, Oxford, 2020, pp. 137-152.

[4] A. de Juan, S.C. Rutan, R. Tauler, Two-way data analysis: multivariate curve resolution-iterative resolution methods, in: S. Brown, R. Tauler, R. Walczak (Eds.), Comprehensive Chemometrics vol. 2, Elsevier, Oxford, 2020, pp. 153-171.

[5] M. Maeder, A.D. Zuberbühler, The resolution of overlapping chromatographic peaks by evolving factor-analysis, Anal. Chim, Acta 181 (1986) 287-291.

[6] H.R. Keller, D.L. Massart, Peak purity control in liquid chromatography with photodiode-array detection by a fixed size moving window evolving factor analysis, Anal. Chim. Acta 246 (1991) 379-390.

[7] O.M. Kvalheim, Y.Z. Liang, Heuristic evolving latent projections: resolving two-way multicomponent data. 1. Selectivity, latent-projective graph, datascope, local rank, and unique resolution, Anal. Chem. 64 (1992) 936-946.

[8] E.R. Malinowski, Window factor analysis: theoretical derivation and application to flow injection analysis data, J. Chemometr. 6 (1992) 29-40.

[9] R. Manne, H. Shen, Y. Liang, Subwindow factor analysis, Chemometr. Intell. Lab. Syst. 45 (1999) 171-176.

[10] W. Windig, J. Guilment, Interactive self-modeling mixture analysis, Anal. Chem. 63 (1991) 1425-1432.

[11] W. Windig, A. Bogomolov, S. Kucheryavski, Two-way data analysis: detection of purest variables, in: S. Brown, R. Tauler, R. Walczak (Eds.), Comprehensive Chemometrics, vol. 2, Elsevier, Oxford, 2020, pp. 107-136.

[12] P.J. Gemperline, A priori estimates of the elution profiles of the pure components in overlapped liquid chromatography peaks using target factor analysis, J. Chem. Inf. Comput. Sci. 24 (1984) 206-212.

[13] B.G.M. Vandeginste, W. Derks, G. Kateman, Multicomponent self-modeling curve resolution in high performance liquid chromatography by iterative target transformation factor analysis, Anal. Chim. Acta 173 (1985) 253-264.

[14] H. Gampp, M. Maeder, C.J. Meyer, A.D. Zuberbühler, Calculation of equilibrium constants from multiwavelength spectroscopic data. III. Model-free analysis of spectrophotometric and ESR titrations, Talanta 32 (1985) 1133-1139.

[15] R. Tauler, E. Casassas, Principal component analysis applied to the study of successive complex formation data in the $\mathrm{Cu}(\mathrm{II})$ ethanolamine systems, J. Chemometr. 3 (1988) 151-161.

[16] R. Tauler, Multivariate curve resolution applied to second order data, Chemometr. Intell. Lab. Syst. 30 (1995) 133-146.

[17] R. Tauler, M. Maeder, A. de Juan, Extended multivariate curve resolution, in: Multiset Data Analysis, in: S. Brown, R. Tauler, R. Walczak (Eds.), Comprehensive Chemometrics, vol. 2, Elsevier, Oxford, 2020, pp. 305-336.

[18] R. Manne, On the resolution problem in hyphenated chromatography chemom, Intell. Lab. Sys. 27 (1995) 89-94.

[19] R. Tauler, A. Smilde, B.R. Kowalski, Selectivity, local rank, three-way data analysis and ambiguity in multivariate curve resolution, J. Chemometr. 9 (1995) $31-58$

[20] J. Ferré, R. Boqué, N.M. Faber, Multiset data analysis: eigenvector-based methods, in: S. Brown, R. Tauler, R. Walczak (Eds.), Comprehensive Chemometrics, vol. 2, Elsevier, Oxford, 2020, pp. 233-266.

[21] G. Tomasi, E. Acar, R. Bro, Multilinear Models, Iterative Methods, in: Comprehensive Chemometrics, S. Brown, R. Tauler, R. Walczak (Eds.) vol. 2, Elsevier, Oxford, 2020, pp. 267-304.

[22] R.A. Harshman, M.E. Lundy, PARAFAC: parallel factor analysis, Comput. Stat. Data Anal. 18 (1994) 39-72.

[23] R. Bro, PARAFAC. Tutorial and applications, Chemometr. Intell. Lab. Syst. 38 (1997) 149-171.

[24] L.R. Tucker, Some mathematical notes on three-mode factor analysis, Psychometrika 31 (1966) 279-311.

[25] A. Smilde, R. Bro, P. Geladi, Multi-way analysis with applications in the chemical sciences, JohnWiley Sons (2005)

[26] E. Sanchez, B.R. Kowalski, Tensorial resolution: a direct trilinear decomposition, J. Chemometr. 4 (1990) 29-45.

[27] Y.L. Xie, P.K. Hopke, P. Paatero, Positive matrix factorization applied to a curve resolution problem, J. Chemometr. 12 (1998) 357-364.

[28] R. Tauler, Interpretation of environmental data using chemometrics, in: D. Barceló (Ed.), Sample Handling and Trace Analysis of Pollutants: Techniques, Applications and Quality Assurance, Elsevier, Amsterdam, 2000, p. 689.
[29] A. de Juan, M. Maeder, M. Martínez, R. Tauler, Combining hard- and softmodelling to solve kinetic problems, Chemometr. Intell. Lab. Syst. 54 (2000) 123-141.

[30] E. Bezemer, S.C. Rutan, Multivariate curve resolution with non-linear fitting of kinetic profiles, Chemometr. Intell. Lab. Syst. 59 (2001) 19-31.

[31] S. Biljsma, A.K. Smilde, Application of curve resolution-based methods to kinetic data, Anal. Chim. Acta 396 (1999) 231-240.

[32] M.C. Antunes, J.E.J. Simao, A.C. Duarte, R. Tauler, Multivariate curve resolution of overlapping voltammetric peaks: quantitative analysis of binary and quaternary metal mixtures, Analyst 127 (2002) 809.

[33] T. Azzouz, R. Tauler, Application of multivariate curve resolution alternating least squares (MCR-ALS) to the quantitative analysis of pharmaceutical and agricultural samples, Talanta 74 (2008) 1201.

[34] R. Tauler, I. Marqués, E. Casassas, Multivariate curve resolution applied to three-way trilinear data: study of a spectrofluorimetric acid-base titration of salicylic acid at three excitation wavelengths, J. Chemometr. 12 (1998) $55-75$.

[35] A. Malik, R. Tauler, Extension and application of multivariate curve resolution-alternating least squares to four-way quadrilinear data-obtained in the investigation of pollution patterns on Yamuna River, India-a case study, Anal. Chim. Acta 794 (2013) 20-28.

[36] M. Alier, M. Felipe, I. Hernández, R. Tauler, Trilinearity and component interaction constraints in the multivariate curve resolution investigation of $\mathrm{NO}$ and $\mathrm{O}_{3}$ pollution in Barcelona, Anal. Bioanal. Chem. 399 (2011) 2015-2029.

[37] P.J. Gemperline, Computation of the range of feasible solutions in selfmodeling curve resolution algorithms, Anal. Chem. 71 (1999) 5398-5404.

[38] R. Tauler, Calculation of maximum and minimum band boundaries of feasible solutions for species profiles obtained by multivariate curve resolution, J. Chemometr. 15 (2001) 627-646.

[39] R. Tauler, M. Maeder, Multivariate curve resolution - error in curve resolution, in: S. Brown, R. Tauler, R. Walczak (Eds.), Comprehensive Chemometrics, vol. 2, Elsevier, Oxford, 2020, pp. 173-197.

[40] A. Golshan, H. Abdollahi, S. Beyramysoltan, M. Maeder, K. Neymeyr, R. Rajkó, M. Sawall, R. Tauler, A review of recent methods for the determination of ranges of feasible solutions resulting from soft modelling analyses of multivariate data. Anal. Chim, Acta 911 (2016) 1-13.

[41] A. de Juan, R. Tauler, R. Dyson, C. Marcolli, M. Rault, M. Maeder, Spectroscopic imaging and chemometrics: a powerful combination for global and local sample analysis, Trac. Trends Anal. Chem. 23 (2004) 70-79.

[42] L. Duponchel, W. Elmi-Rayaleh, C. Ruckebusch, J.P. Huvenne, Multivariate curve resolution methods in imaging spectroscopy: influence of extraction methods and instrumental perturbations, J. Chem. Inf. Comput. Sci. 43 (2003) 2057-2067.

[43] J.M. Amigo, J. Cruz, M. Bautista, S. Maspoch, J. Coello, M. Blanco, Study of pharmaceutical samples by NIR chemical-image and multivariate analysis. TrAC Trends in Anal, Inside Chem. 27 (2008) 696-713.

[44] S.E. Porter, D.R. Stoll, S.C. Rutan, P.W. Carr, J.D. Cohen, Analysis of four-way two-dimensional liquid chromatography-diode array data: application to metabolomics, Anal. Chem. 78 (2006) 5559-5569.

[45] K.M. Pierce, J.C. Hoggard, R.E. Mohler, R.E. Synovec, Recent advancements in comprehensive two-dimensional separations with chemometrics, J. Chromatogr. A 1184 (2008) 341-352.

[46] H. Parastar, R. Tauler, Multivariate curve resolution of hyphenated and multidimensional chromatographic measurements: a new insight to address current chromatographic challenges, Anal. Chem. 86 (2014) 286-297.

[47] M. Alier, R. Tauler, Multivariate curve resolution of incomplete data multisets, Chemometr. Intell. Lab. Syst. 127 (2013) 17-28.

[48] A. Gómez-Sánchez, M. Marro, M. Marsal, P. Loza-Alvarez, A. de Juan, 3D and 4D image fusion: coping with differences in spectroscopic modes among hyperspectral images, Anal. Chem. (2020), https://doi.org/10.1021/ acs analchem 0 c00780.

[49] S. Hugelier, O. Devos, C. Ruckebusch, On the implementation of spatial constraints in multivariate curve resolution alternating least squares for hyperspectral image analysis, J. Chemometr. 29 (2015) 557-561.

[50] S. Hugelier, S. Piqueras, C. Bedia, A. de Juan, C. Ruckebusch, Application of a sparseness constraint in multivariate curve resolution-alternating least squares, Anal. Chim. Acta 1000 (2018) 100-108.

[51] A. de Juan, M. Maeder, T. Hancewicz, R. Tauler, Use of local rank-based spatial information for resolution of spectroscopic images, J. Chemom. 22 (2008) 291-298.

[52] M. Sawall, H. Schröder, D. Meinhardt, K. Neymeyr, On the ambiguity underlying Multivariate Curve Resolution models, in: S. Brown, R. Tauler, R. Walczak (Eds.), Comprehensive Chemometrics, vol. 2, Elsevier, Oxford, 2020, pp. 199-231.

[53] E. Gorrochategui, J. Jaumot, S. Lacorte, R. Tauler, Data analysis strategies for targeted and untargeted LC-MS metabolomic studies: overview and workflow. TrAC Trends in Anal, Inside Chem. 82 (2016) 425-442.

[54] P. Jonsson, E.S. Johansson, A. Wuolikainen, J. Lindberg, I. Schuppe-Koistinen, M. Kusano, M. Sjöström, J. Trygg, T. Moritz, H. Antti, Predictive metabolite profiling applying hierarchical multivariate curve resolution to GC- MS data a potential tool for multi-parametric diagnosis, J. Proteome Res. 5 (2006) 1407-1414.

[55] J.C. Hamilton, P.J. Gemperline, Mixture analysis using factor analysis. II: selfmodeling curve resolution, J. Chemometr. 4 (1990) 1-13. 
[56] A. de Juan, R. Tauler, Chemometrics applied to unravel multicomponent processes and mixtures. Revisiting latest trends in multivariate resolution, Anal. Chim. Acta 500 (2003) 195-210.

[57] J.H. Jiang, Y. Liang, Y. Ozaki, Principles and methodologies in self-modeling curve resolution, Chemometr. Intell. Lab. Syst. 71 (2004) 1-12.

[58] A. de Juan, R. Tauler, Multivariate curve resolution (MCR) from 2000: progress in concepts and applications, Crit. Rev. Anal. Chem. 36 (2006) 163-176.

[59] C. Ruckebusch, L. Blanchet, Multivariate curve resolution: a review of advanced and tailored applications and challenges, Anal. Chim. Acta 765 (2013) 28-36.

[60] A. de Juan, J. Jaumot, R. Tauler, Multivariate Curve Resolution (MCR). Solving the mixture analysis problem, Analyt. Meth. 6 (2014) 4964-4976.

[61] R. Bro, S. de Jong, A fast non-negativity-constrained least squares algorithm, J. Chemometr. 11 (1997) 393-401.

[62] R. Bro, N.D. Sidiropoulos, Least squares algorithms under unimodality and non-negativity constraints, J. Chemometr. 12 (1998) 223-247.

[63] J.M. Amigo, A. de Juan, J. Coello, S. Maspoch, A mixed hard-and softmodelling approach for the quantitative determination of oxipurines and uric acid in human urine. Anal. Chim, Acta 567 (2006) 236-244.

[64] L. Blanchet, A. Mezzetti, C. Ruckebusch, J.P. Huvenne, A. de Juan, Multivariate curve resolution of rapid-scan FTIR difference spectra of quinone photoreduction in bacterial photosynthetic membranes, Anal. Bioanal. Chem. 387 (2007) 1863-1873.

[65] S. Mas, R. Bendoula, G. Agoda-Tandjawa, A. de Juan, J.M. Roger, Study of time-dependent structural changes of laponite colloidal system by means of near-infrared spectroscopy and hybrid hard-and soft-modelling multivariate curve resolution-alternating least squares, Chemometr. Intell. Lab. Syst. 142 (2015) 285-292.

[66] J. Diewok, A. de Juan, M. Maeder, R. Tauler, B. Lendl, Application of a combination of hard and soft modeling for equilibrium systems to the quantitative analysis of pH-modulated mixture samples, Anal. Chem. 75 (2003) 641-647.

[67] C. Ruckebusch, M. Sliwa, P.D. Pernot, A. de Juan, R. Tauler, Comprehensive data analysis of femtosecond transient absorption spectra: a review, J. Photochem. Photobiol. C Photochem. Rev. 13 (2012) 1-27.

[68] L. Blanchet, C. Ruckebusch, J.P. Huvenne, A. de Juan, Hybrid hard-and softmodeling applied to difference spectra, Chemometr. Intell. Lab. Syst. 89 (2007) 26-35.

[69] S. Mas, R. Tauler, A. de Juan, Chromatographic and spectroscopic data fusion analysis for interpretation of photodegradation processes, J. Chromatogr. A 1218 (2011) 9260-9268.

[70] M. De Luca, G. Ragno, G. Ioele, R. Tauler, Multivariate curve resolution of incomplete fused multiset data from chromatographic and spectrophotometric analyses for drug photostability studies, Anal. Chim. Acta 837 (2014) $31-37$.

[71] S. Mas, C. Miguel, M.J. Melo, J.A. Lopes, A. de Juan, Screening and quantification of proteinaceous binders in medieval paints based on $\mu$-Fourier transform infrared spectroscopy and multivariate curve resolution alternating least squares, Chemometr. Intell. Lab. Syst. 134 (2014) 148-157.

[72] S.E. Richards, E. Becker, R. Tauler, A.D. Walmsley, A novel approach to the quantification of industrial mixtures from the Vinyl Acetate Monomer (VAM) process using Near Infrared spectroscopic data and a Quantitative Self Modeling Curve Resolution (SMCR) methodology, Chemom. Intell. Lab. Sys. 94 (2008) 9-18.

[73] L.B. Lyndgaard, F. van den Berg, A. de Juan, Quantification of paracetamol through tablet blister packages by Raman spectroscopy and multivariate curve resolution-alternating least squares, Chemometr. Intell. Lab. Syst. 125 (2013) 58-66.

[74] H.C. Goicoechea, A.C. Olivieri, R. Tauler, Application of the correlation constrained multivariate curve resolution alternating least-squares method for analyte quantitation in the presence of unexpected interferences using firstorder instrumental data, Analyst 135 (2010) 636-642.

[75] R.R. de Oliveira, K.M.G. de Lima, R. Tauler, A. de Juan, Application of correlation constrained multivariate curve resolution alternating least-squares methods for determination of compounds of interest in biodiesel blends using NIR and UV-visible spectroscopic data, Talanta 125 (2014) 233-241.

[76] M. Akbari Lakeh, H. Abdollahi, P.J. Gemperline, Soft known-value constraints for improved quantitation in multivariate curve resolution, Anal. Chim. Acta 1105 (2020) 64-73.

[77] R. Tauler, A. de Juan, Multivariate curve resolution for quantitative analysis, in: Data Handling in Science and Technology, vol. 29, Elsevier, 2015, pp. 247-292.

[78] A.C. de Oliveira Neves, R. Tauler, K.M.G. de Lima, Area correlation constraint for the MCR-ALS quantification of cholesterol using EEM fluorescence data: a new approach. Anal. Chim, Acta 937 (2016) 21-28.

[79] M. Ghaffari, H. Abdollahi, A conceptual view to the area correlation constraint in multivariate curve resolution, Chemom. Intell. Lab. Sys. 189 (2019) $121-129$.

[80] M. Bayat, M. Marín-García, J.B. Ghasemi, R. Tauler, Application of the area correlation constraint in the MCR-ALS quantitative analysis of complex mixture samples, Anal. Chim. Acta 1113 (2020) 52-65.

[81] D.W. Cook, S.C. Rutan, Analysis of liquid chromatography-mass spectrometry data with an elastic net multivariate curve resolution strategy for sparse spectral recovery, Anal. Chem. 89 (2017) 8405-8412.

[82] N. Omidikia, M. Ghaffari, R. Rajkó, Sparse non-negative multivariate curve resolution: L0, L1, or L2 norms? Chemom, Intell. Lab. Sys. 199 (2020), 103969.

[83] W. Windig, M.R. Keenan, Angle-constrained alternating least squares, Appl, Spectrosc. 65 (2011) 349-357.

[84] W. Windig, J.M. Shaver, M.R. Keenan, B.M. Wise, Simplification of alternating least squares solutions with contrast enhancement, Chemometr. Intell. Lab. Syst. 117 (2012) 159-168.

[85] C.G. Bertinetto, A. de Juan, Systematic comparison and potential combination between multivariate curve resolution-alternating least squares (MCR-ALS) and band-target entropy minimization (BTEM), J. Chemometr. 32 (2018) e3000.

[86] D.N. Rutledge, D. Jouan-Rimbaud Bouveresse, Independent components analysis with the JADE algorithm, Trac. Trends Anal. Chem. 50 (2013) 22-32.

[87] H. Parastar, M. Jalali-Heravi, R. Tauler, Is independent component analysis appropriate for multivariate resolution in analytical chemistry? Trends Anal. Chem. 31 (2012) 134-143.

[88] S. Hugelier, R. Vitale, C. Ruckebusch, Edge-preserving image smoothing constraint in multivariate curve resolution-alternating least squares (MCRALS) of hyperspectral data, Appl. Spectrosc. 72 (2018) 420-431.

[89] P. Firmani, S. Hugelier, F. Marini, C. Ruckebusch, MCR-ALS of hyperspectral images with spatio-spectral fuzzy clustering constraint, Chemometr. Intell. Lab. Syst. 179 (2018) 85-91.

[90] A. de Juan, R. Tauler, Multivariate curve resolution-alternating least squares for spectroscopic data, in: Data Handling in Science and Technology, vol. 30, Elsevier, 2016, pp. 5-51.

[91] J. Jaumot, N. Escaja, R. Gargallo, C. González, E. Pedroso, R. Tauler, Multivariate curve resolution: a powerful tool for the analysis of conformational transitions in nucleic acids, Nucleic Acids Res. 30 (2002) e92-e92.

[92] A. de Juan, S. Mas, M. Maeder, R. Tauler, A perspective on modeling evolution, J. Chemometr. 34 (2020) e3205.

[93] R. Tauler, D. Barceló, Multivariate curve resolution applied to liquidchromatography diode-array detection. TrAC- Trends Anal, Inside Chem. 12 (1993) 319-327.

[94] E. Peré-Trepat, S. Lacorte, R. Tauler, Alternative calibration approaches for LC-MS quantitative determination of coeluted compounds in complex environmental mixtures using multivariate curve resolution, Anal. Chim. Acta 595 (2007) 228-237.

[95] J. Saurina, C. Leal, R. Compañó, M. Granados, M.D. Prat, R. Tauler, Estimation of figures of merit using univariate statistics for quantitative second-order multivariate curve resolution, Anal. Chim. Acta 432 (2001) 241-251.

[96] M.C. Ortiz, L. Sarabia, Quantitative determination in chromatographic analysis based on n-way calibration strategies, J. Chromatogr. A 1158 (2007) 94-110.

[97] J. Jaumot, R. Gargallo, R. Tauler, Noise propagation and error estimations in multivariate curve resolution alternating least squares using resampling methods, J. Chemometr. 18 (2004) 327-340.

[98] A.C. Olivieri, N.M. Faber, A closed-form expression for computing the sensitivity in second-order bilinear calibration, J. Chemometr. 19 (2005) 583-592.

[99] A.C. Olivieri, Computing sensitivity and selectivity in parallel factor analysis and related multiway techniques: the need for further developments in net analyte signal theory, Anal. Chem. 77 (2005) 4936-4946.

[100] M.C. Bauza, G.A. Ibañez, R. Tauler, A.C. Olivieri. Sensitivity equation for quantitative analysis with multivariate curve resolution-alternating leastsquares: theoretical and experimental approach, Anal. Chem. 84 (20) (2012) 8697-8706.

[101] M. Marín-García, R. Tauler, Chemometrics characterization of the Llobregat river dissolved organic matter, Chemometr. Intell. Lab. Syst. (2020), 104018.

[102] M.D. Carabajal, J.A. Arancibia, G.M. Escandar, Excitation-emission fluorescence-kinetic third-order/four-way data: determination of bisphenol A and nonylphenol in food-contact plastics, Talanta 197 (2019) 348-355.

[103] E. Tavakkoli, H. Abdollahi, P.J. Gemperline, Soft-trilinear constraints for improved quantitation in multivariate curve resolution, Analyst 145 (2020) 223-232.

[104] A. Malik, R. Tauler, Performance and validation of MCR-ALS with quadrilinear constraint in the analysis of noisy datasets, Chemometr. Intell. Lab. Syst. 135 (2014) 223-234.

[105] R. Tauler, Multivariate Curve resolution of multiway data using the multilinearity constraint, J. Chemometr. (2020), https://doi.org/10.1002/cem.3279.

[106] M. Terrado, D. Barceló, R. Tauler, Quality assessment of the multivariate curve resolution alternating least squares method for the investigation of environmental pollution patterns in surface water, Environ. Sci. Technol. 43 (2009) 5321-5326.

[107] S. Elcoroaristizábal, A. de Juan, J.A. García, N. Durana, L. Alonso, Comparison of second-order multivariate methods for screening and determination of PAHs by total fluorescence spectroscopy, Chemometr. Intell. Lab. Syst. 132 (2014) 63-74.

[108] S. Cavanillas, J.M. Díaz-Cruz, C. Ariño, M. Esteban, Parametric signal fitting by Gaussian peak adjustment: a new multivariate curve resolution method for non-bilinear voltammetric measurements, Anal. Chim. Acta 689 (2011) $198-205$.

[109] J. Jaumot, M. Vives, R. Gargallo, R. Tauler, Multivariate resolution of NMR labile signals by means of hard-and soft-modelling methods. Analytica Chim, Acta 490 (2003) 253-264.

[110] B. Debus, M. Sliwa, H. Miyasaka, J. Abe, C. Ruckebusch, Multivariate curve 
resolution-alternating least squares to cope with deviations from data bilinearity in ultrafast time-resolved spectroscopy, Chemometr. Intell. Lab. Syst. 128 (2013) 101-110.

[111] A.L. Pomerantsev, Y.V. Zontov, O.Y. Rodionova, Nonlinear multivariate curve resolution alternating least squares (NL-MCR-ALS), J. Chemometr. 28 (2014) 740-748.

[112] S. Piqueras, C. Bedia, C. Beleites, C. Krafft, J. Popp, M. Maeder, R. Tauler, A. de Juan, Handling different spatial resolutions in image fusion by multivariate curve resolution-alternating least squares for incomplete image multisets, Anal. Chem. 90 (2018) 6757-6765.

[113] E. Acar, M.A. Rasmussen, F. Savorani, T. Næs, R. Bro, Understanding data fusion within the framework of coupled matrix and tensor factorizations, Chemometr. Intell. Lab. Syst. 129 (2013) 53-63.

[114] A.C. Olivieri, Second-order multivariate calibration with the extended bilinear model: effect of initialization, constraints, and composition of the calibration set on the extent of rotational ambiguity, J. Chemometr. 34 (2020) e3130.

[115] M. Sawall, C. Kubis, H. Schröder, D. Meinhardt, D. Selent, R. Franke, A. Brächer, A. Börner, K. Neymeyr, Multivariate curve resolution methods and the design of experiments, J. Chemometr. 34 (2020) e3159.

[116] M. Akbari, H. Abdollahi, Investigation and visualization of resolution theorems in self modeling curve resolution (SMCR) methods, J. Chemometr. 27 (2013) 278-286.

[117] R. Rajko, Natural duality in minimal constrained self-modeling curve resolution, J. Chemometr. 20 (2006) 164-169.

[118] A.C. Olivieri, R. Tauler, The effect of data matrix augmentation and constraints in extended multivariate curve resolution-alternating least squares, J. Chemometr. 31 (2017) e2875.

[119] J. Jaumot, R. Tauler, MCR-BANDS: a user friendly MATLAB program for the evaluation of rotation ambiguities in Multivariate Curve Resolution, Chemom. Intell. Lab. Sys. 103 (2010) 96-107.

[120] J. Jaumot, A. de Juan, R. Tauler, MCR-ALS GUI 2.0: new features and applications, Chemometr. Intell. Lab. Syst. 140 (2015) 1-12.

[121] X. Zhang, Z. Zhang, R. Tauler, Evaluation of the extension of rotation ambiguity associated to multivariate curve resolution solutions by the application of the MCR-BANDS method, Talanta 202 (2019) 554-564.

[122] K. Neymeyr, A. Golshan, K. Engel, R. Tauler, M. Sawall, Does the signal contribution function attain its extrema on the boundary of the area of feasible solutions? Chemometr. Intell. Lab. Syst. 196 (2020), 103887.

[123] R. Rajkó, K. István, Analytical solution for determining feasible regions of self-modeling curve resolution (SMCR) method based on computational geometry, J. Chemometr.: J. Chemometr. Soc. 19 (2005) 448-463.

[124] H. Abdollahi, M. Maeder, R. Tauler, Calculation and meaning of feasible band boundaries in multivariate curve resolution of a two-component system, Anal. Chem. 81 (2009) 2115-2122.

[125] A. Golshan, H. Abdollahi, M. Maeder, Resolution of rotational ambiguity for three-component systems, Anal. Chem. 83 (2011) 836-841.

[126] A. Golshan, M. Maeder, H. Abdollahi, Determination and visualization of rotational ambiguity in four-component systems, Anal. Chim. Acta 796 (2013) 20-26.

[127] M. Sawall, C. Kubis, D. Selent, A. Boerner, K. Neymeyr, A fast polygon inflation algorithm to compute the area of feasible solutions for three-component systems. I: concepts and applications, J. Chemometr. 27 (2013) 106-116.

[128] M. Sawall, A. Jürß, H. Schröder, K. Neymeyr, On the analysis and computation of the area of feasible solutions for two-, three-, and four-component systems, in: Data Handling in Science and Technology, vol. 30, Elsevier, 2016, pp. $135-184$.

[129] M. Sawall, K. Neymeyr, A fast polygon inflation algorithm to compute the area of feasible solutions for three-component systems. II: theoretical foundation, inverse polygon inflation, and FAC-PACK implementation, J. Chemometr. 28 (2014) 633-644.

[130] S. Beyramysoltan, R. Rajkó, H. Abdollahi, Investigation of the equality constraint effect on the reduction of the rotational ambiguity in threecomponent system using a novel grid search method, Anal. Chim. Acta 791 (2013) 25-35.

[131] M. Akbari Lakeh, H. Abdollahi, Known-value constraint in multivariate curve resolution, Anal. Chim. Acta 1030 (2018) 42-51.

[132] A. Golshan, H. Abdollahi, M. Maeder, The reduction of rotational ambiguity in soft-modeling by introducing hard models. Anal. Chim, Acta 709 (2012) $32-40$.

[133] M. Ghaffari, S. Hugelier, L. Duponchel, H. Abdollahi, C. Ruckebusch, Effect of image processing constraints on the extent of rotational ambiguity in MCRALS of hyperspectral images, Anal. Chim. Acta 1052 (2019) 27-36.

[134] R.B. Pellegrino Vidal, A.C. Olivieri, R. Tauler, Quantifying the prediction error in analytical multivariate curve resolution studies of multicomponent systems, Anal. Chem. 90 (2018) 7040-7047.

[135] R.B. Pellegrino Vidal, A.C. Olivieri, A new parameter for measuring the prediction uncertainty produced by rotational ambiguity in second-order calibration with multivariate curve resolution, Anal. Chem. (2020), https:// doi.org/10.1021/acs.analchem.0c01395.

[136] M.N. Leger, P.D. Wentzell, Dynamic Monte Carlo self-modeling curve resolution method for multicomponent mixtures, Chemometr. Intell. Lab. Syst. 62 (2002) 171-188.

[137] M. Dadashi, H. Abdollahi, R. Tauler, Error propagation along the different regions of multivariate curve resolution feasible solutions, Chemometr.
Intell. Lab. Syst. 162 (2017) 203-213.

[138] P.D. Wentzell, Other topics in soft-modeling: maximum likelihood-based soft-modeling methods, in: S. Brown, R. Tauler, R. Walczak (Eds.), Comprehensive Chemometrics, vol. 2, Elsevier, Oxford, 2020, pp. 399-439.

[139] P.D. Wentzell, T.K. Karakach, S. Roy, M.J. Martinez, C.P. Allen, M. WernerWashburne, Multivariate curve resolution of time course microarray data, BMC Bioinform. 7 (1) (2006) 343.

[140] R. Tauler, M. Viana, X. Querol, A. Alastuey, R.M. Flight, P.D. Wentzell, P.K. Hopke, Comparison of the results obtained by four receptor modelling methods in aerosol source apportionment studies, Atmos. Environ. 43 (2009) 3989-3997.

[141] M. Dadashi, H. Abdollahi, R. Tauler, Maximum likelihood principal component analysis as initial projection step in multivariate curve resolution analysis of noisy data, Chemometr. Intell. Lab. Syst. 118 (2012) 33-40.

[142] P.K. Hopke, Review of receptor modeling methods for source apportionment J. Air Waste Manag. Assoc. 66 (2016) 237-259.

[143] P. Paatero, The multilinear engine-a table-driven, least squares program for solving multilinear problems, including the n-way parallel factor analysis model, J. Comput. Graph Stat. 8 (1999) 854-888.

[144] F.B. Lavoie, N. Braidy, R. Gosselin, Including noise characteristics in MCR to improve mapping and component extraction from spectral images, Chemometr. Intell. Lab. Syst. 153 (2016) 40-50.

[145] E.S. Park, P.K. Hopke, I. Kim, S. Tan, C.H. Spiegelman, Bayesian spatial multivariate receptor modeling for multisite multipollutant data. Technometrics 60 (3) (2018) 306-318.

[146] J. Jaumot, B. Piña, R. Tauler, Application of multivariate curve resolution to the analysis of yeast genome-wide screens, Chemometr. Intell. Lab. Syst. 104 (2010) 53-64.

[147] L. Blanchet, J. Réhault, C. Ruckebusch, J.P. Huvenne, R. Tauler, A. de Juan, Chemometrics description of measurement error structure: study of an ultrafast absorption spectroscopy experiment, Anal. Chim. Acta 642 (2009) 19-26.

[148] A. Martins, J. Coddington, G. Van der Snickt, B. Van Driel, C. McGlinchey, D. Dahlberg, K. Janssens, J. Dik, Jackson Pollock's Number 1A, 1948: a noninvasive study using macro-X-ray fluorescence mapping (MA-XRF) and multivariate curve resolution-alternating least squares (MCR-ALS) analysis, Heritage Sci. 4 (2016) 1-13.

[149] M. Maeder, Y.M. Neuhold, G. Puxty, P.J. Gemperline, Advances in the modelling and analysis of complex and industrial processes, Chemometr. Intell. Lab. Syst. 82 (2006) 75-82.

[150] A. de Juan, R. Tauler, Data fusion by multivariate curve resolution, in: Data Handling in Science and Technology, vol. 31, Elsevier, 2019, pp. 205-233.

[151] S. Mas, A. de Juan, R. Tauler, A.C. Olivieri, G.M. Escandar, Application of chemometric methods to environmental analysis of organic pollutants: a review, Talanta 80 (2010) 1052-1067.

[152] M. Marín-García, G. Ioele, H. Franquet-Griell, S. Lacorte, G. Ragno, R. Tauler, Investigation of the photodegradation profile of tamoxifen using spectroscopic and chromatographic analysis and multivariate curve resolution, Chemometr. Intell. Lab. Syst. 174 (2018) 128-141.

[153] S. Grassi, C. Alamprese, V. Bono, E. Casiraghi, J.M. Amigo, Modelling milk lactic acid fermentation using multivariate curve resolution-alternating least squares (MCR-ALS). Food and Bioproc, Technol. 7 (2014) 1819-1829.

[154] W. Kessler, R.W. Kessler, Multivariate curve resolution: a method of evaluating the kinetics of biotechnological reactions, Anal. Bioanal. Chem. 384 (2006) 1087-1095.

[155] J. Jaumot, B. Igne, C.A. Anderson, J.K. Drennen, A. de Juan, Blending process modeling and control by multivariate curve resolution, Talanta 117 (2013) 492-504.

[156] R. Gargallo, Hard/soft hybrid modeling of temperature-induced unfolding processes involving G-quadruplex and i-motif nucleic acid structures, Anal. Biochem 466 (2014) 4-15.

[157] S. Benabou, C. Ruckebusch, M. Sliwa, A. Aviñó, R. Eritja, R. Gargallo, A de Juan, Study of conformational transitions of i-motif DNA using time-resolved fluorescence and multivariate analysis methods, Nucleic Acids Res. 47 (2019) 6590-6605.

[158] P. Cutler, P.J. Gemperline, A. de Juan, Experimental monitoring and data analysis tools for protein folding: study of steady-state evolution and modeling of kinetic transients by multitechnique and multiexperiment data fusion, Anal. Chim. Acta 632 (2009) 52-62.

[159] R. Tautenhahn, C. Bottcher, S. Neumann, Highly sensitive feature detection for high resolution LC/MS, BMC Bioinf. 9 (2008) 504.

[160] E. Gorrochategui, J. Jaumot, R. Tauler, ROIMCR: a powerful analysis strategy for LC-MS metabolomic datasets, BMC Bioinf. 20 (2019) 256.

[161] C. Bedia, Metabolomics applications in environmental research, in: Data Analysis for Omic Sciences: Methods and Applications, vol. 82, Elsevier, 2018.

[162] M.D. Carabajal, J.A. Arancibia, G.M. Escandar, Multivariate curve resolution strategy for non-quadrilinear type 4 third-order/four-way liquid chromatography excitation-emission fluorescence matrix data, Talanta 189 (2018) 509-516.

[163] M.R. Alcaraz, O. Monago-Maraña, H.C. Goicoechea, A. Muñoz de la Peña, Four- and five-way excitation-emission luminescence-based data acquisition and modeling for analytical applications. A review, Anal. Chim, Acta 1083 (2019) 41-57.

[164] E. Peré-Trepat, R. Tauler, Analysis of environmental samples by application 
of multivariate curve resolution on fused high-performance liquid chromatography-diode array detection mass spectrometry data, J. Chromatogr. A 1131 (2006) 85-96.

[165] G. Tomasi, F. Van Den Berg, C. Andersson, Correlation optimized warping and dynamic time warping as preprocessing methods for chromatographic data, J. Chemometr. 18 (2004) 231-241.

[166] G. Tomasi, F. Savorani, S.B. Engelsen, Icoshift: an effective tool for the alignment of chromatographic data, J. Chromatogr. A 1218 (2011) $7832-7840$.

[167] G.M. Escandar, A.C. Olivieri, Multi-way chromatographic calibration-a review, J. Chromatogr. A 1587 (2019) 2-13.

[168] R. Tauler, P. Paatero, R.C. Henry, P.K. Hopke, Identification, resolution and apportionment of contamination sources: paatero, Pentti, in: J. Anthony, Jakeman, A. Alexey, Voinov, E. Andrea, Rizzoli, H. Serena, Chen (Eds.), Environmental Modelling, Software and Decision Support, 3, Elsevier, 2008, pp. 269-284.

[169] E.S. Park, R. Tauler, Bayesian methods for factor Analysis in chemometrics, in: S. Brown, R. Tauler, R. Walczak (Eds.), Comprehensive Chemometrics, vol. 2, Elsevier, Oxford, 2020, pp. 355-369.

[170] M. Terrado, M. Kuster, D. Raldúa, M.L. de Alda, D. Barceló, R. Tauler, Use of chemometric and geostatistical methods to evaluate pesticide pollution in the irrigation and drainage channels of the Ebro river delta during the ricegrowing season, Anal. Bioanal. Chem. 387 (2007) 1479-1488.

[171] M. Terrado, M.P. Lavigne, S. Tremblay, S. Duchesne, J.P. Villeneuve, A.N. Rousseau, D. Barceló, R. Tauler, Distribution and assessment of surface water contamination by application of chemometric and deterministic models, J. Hydrol 369 (2009) 416-426.

[172] A. de Juan, Multivariate curve resolution for hyperspectral image analysis, in: Data Handling in Science and Technology, vol. 32, Elsevier, 2020, pp. $115-150$.

[173] M. Pérez-Cova, R. Tauler, J. Jaumot, Chemometrics in comprehensive twodimensional liquid chromatography: a study of the data structure and its multilinear behavior, Chemometr. Intell. Lab. Syst. (2020), 104009.

[174] J.M. Bioucas-Dias, A. Plaza, N. Dobigeon, M. Parente, Q. Du, P. Gader, J. Chanussot, Hyperspectral unmixing overview: geometrical, statistical, and sparse regression-based approaches. IEEE j. sel. topics appl. earth obs, Remote Sens. 5 (2012) (2012) 354-379.

[175] X. Zhang, R. Tauler, Application of multivariate curve resolution alternating least squares (MCR-ALS) to remote sensing hyperspectral imaging, Anal. Chim. Acta 762 (2013) 25-38.

[176] X. Zhang, A. de Juan, R. Tauler, Local rank-based spatial information for improvement of remote sensing hyperspectral imaging resolution, Talanta 146 (2016) 1-9.

[177] G.L. Alexandrino, M.R. Khorasani, J.M. Amigo, J. Rantanen, R.J. Poppi, Monitoring of multiple solid-state transformations at tablet surfaces using multiseries near-infrared hyperspectral imaging and multivariate curve resolution, Eur. J. Pharm. Biopharm. 93 (2015) 224-230.

[178] S. Piqueras, L. Duponchel, R. Tauler, A. de Juan, Monitoring polymorphic transformations by using in situ Raman hyperspectral imaging and image multiset analysis, Anal. Chim. Acta 819 (2014) 15-25.

[179] F.D.S.L. Borba, T. Jawhari, R.S. Honorato, A. de Juan, Confocal Raman imaging and chemometrics applied to solve forensic document examination involving crossed lines and obliteration cases by a depth profiling study, Analyst 142 (7) (2017) 1106-1118.

[180] S. Piqueras, J. Burger, R. Tauler, A. de Juan, Relevant aspects of quantification and sample heterogeneity in hyperspectral image resolution, Chemometr. Intell. Lab. Syst. 117 (2012) 169-182.

[181] A. de Juan, A. Gowen, L. Duponchel, C. Ruckebusch, Image fusion, in: Data Handling in Science and Technology, vol. 31, Elsevier, 2019, pp. 311-344.

[182] J. Fortuna, H. Martens, T.A. Johansen, Multivariate image fusion: a pipeline for hyperspectral data enhancement, Chemometr. Intell. Lab. Syst. (2020), 104097.

[183] R.A. Schowengerdt, Spatial transforms, in: R.A. Schowengerdt (Ed.), Remote Sensing Modelling Methods for Image Processing, 3th ed, 2006, pp. 229-282.

[184] S. Piqueras, M. Maeder, R. Tauler, A. de Juan, A new matching image preprocessing for image data fusion, Chemom. Intell. Lab. Sys. 164 (2017) (2017) $32-42$.

[185] C. Bedia, À. Sierra, R. Tauler. Application of chemometric methods to the analysis of multimodal chemical images of biological tissues, Anal. Bioanal. Chem. 412 (2020) 5179-5190.

[186] S. Mas, A. Torro, N. Bec, L. Fernández, G. Erschov, C. Gongora, C. Larroque, P. Martineau, A. de Juan, S. Marco, Use of physiological information based on grayscale images to improve mass spectrometry imaging data analysis from biological tissues. Analytica Chim, Acta 1074 (2019) 69-79.

[187] S. Piqueras, C. Krafft, C. Beleites, K. Egodage, F. Von Eggeling, O. GuntinasLichius, J. Popp, R. Tauler, A. de Juan, Combining multiset resolution and segmentation for hyperspectral image analysis of biological tissues, Anal. Chim. Acta 881 (2015) 24-36.

[188] S. Piqueras, L. Duponchel, M. Offroy, F. Jamme, R. Tauler, A. de Juan, Chemometric strategies to unmix information and increase the spatial description of hyperspectral images: a single-cell case study, Anal. Chem. 85 (2013) 6303-6311.

[189] C. Bedia, R. Tauler, J. Jaumot, Compression strategies for the chemometric analysis of mass spectrometry imaging data, J. Chemometr. 30 (2016) (2016) 575-588.
[190] C. Bedia, R. Tauler, J. Jaumot, Analysis of multiple mass spectrometry images from different Phaseolus vulgaris samples by multivariate curve resolution, Talanta 175 (2017) 557-565.

[191] M. Ghaffari, N. Omidikia, C. Ruckebusch, Essential spectral pixels for multivariate curve resolution of chemical images, Anal. Chem. 91 (2019) 10943-10948.

[192] A. Nardecchia, L. Duponchel, Randomised SIMPLISMA: using a dictionary of initial estimates for spectral unmixing in the framework of chemical imaging, Talanta (2020), 121024.

[193] V.G. van Mispelaar, A.C. Tas, A.K. Smilde, P.J. Schoenmakers, A.C. van Asten, Quantitative analysis of target components by comprehensive twodimensional gas chromatography, J. Chromatogr. A 1019 (2003) 15-29.

[194] N.E. Watson, W.C. Siegler, J.C. Hoggard, R.E. Synovec, Comprehensive threedimensional gas chromatography with parallel factor analysis, Anal. Chem. 79 (2007) 8270-8280.

[195] M. Navarro-Reig, J. Jaumot, A. Baglai, G. Vivó-Truyols, P.J. Schoenmakers, R. Tauler, Untargeted comprehensive two-dimensional liquid chromatography coupled with high-resolution mass spectrometry analysis of rice metabolome using multivariate curve resolution, Anal. Chem. 89 (2017) $7675-7683$.

[196] N.G.S. Mogollon, F.A. de Lima Ribeiro, M.M. Lopez, L.W. Hantao, R.J. Poppi, F. Augusto, Quantitative analysis of biodiesel in blends of biodiesel and conventional diesel by comprehensive two-dimensional gas chromatography and multivariate curve resolution, Anal. Chim. Acta 796 (2013) $130-136$.

[197] J. Omar, M. Olivares, J.M. Amigo, N. Etxebarria, Resolution of co-eluting compounds of Cannabis sativa in comprehensive two-dimensional gas chromatography/mass spectrometry detection with multivariate curve resolution-alternating least squares, Talanta 121 (2014) 273-280.

[198] T. Skov, J.C. Hoggard, R. Bro, R.E. Synovec, Handling within run retention time shifts in two-dimensional chromatography data using shift correction and modeling, J. Chromatogr. A 1216 (2009) 4020-4029.

[199] J. Vial, H. Noçairi, P. Sassiat, S. Mallipatu, G. Cognon, D. Thiébaut, B. Teillet, D.R. Rutledge, Combination of dynamic time warping and multivariate analysis for the comparison of comprehensive two-dimensional gas chromatograms: application to plant extracts, J. Chromatogr. A 1216 (14) (2009) 2866-2872, 2009.

[200] D.W. Cook, S.C. Rutan, D.R. Stoll, P.W. Carr, Two-dimensional assisted liquid chromatography-a chemometric approach to improve accuracy and precision of quantitation in liquid chromatography using 2D separation, dual detectors, and multivariate curve resolution, Anal. Chim. Acta 859 (2015) 87-95.

[201] D.M. Haaland, J.A. Timlin, M.B. Sinclair, M.H. Van Benthem, M.J. Martinez, A.D. Aragon, M. Werner-Washburne, Multivariate curve resolution for hyperspectral image analysis: applications to microarray technology, in: Spectral Imaging: Instrumentation, Applications, and Analysis II, vol. 4959, International Society for Optics and Photonics, 2003, pp. 55-66.

[202] J. Jaumot, R. Tauler, R. Gargallo, Exploratory data analysis of DNA microarrays by multivariate curve resolution, Anal. Biochem. 358 (2006) 76-89.

[203] A. Smirnov, Y. Qiu, W. Jia, D.I. Walker, D.P. Jones, X. Du, ADAP-GC 4.0: application of clustering-assisted multivariate curve resolution to spectral deconvolution of gas chromatography-mass spectrometry metabolomics data, Anal. Chem. 91 (2019) 9069-9077.

[204] E. Gorrochategui, J. Jaumot, R. Tauler, A protocol for LC-MS metabolomic data processing using chemometric tools, Protocol Exchange (2015), https:// doi.org/10.1038/protex.2015.12.

[205] R. Wehrens, E. Carvalho, P.D. Fraser, Metabolite profiling in LC-DAD using multivariate curve resolution: the alsace package for R, Metabolomics 11 (2015) 143-154.

[206] E. Ortiz-Villanueva, F. Benavente, B. Piña, V. Sanz-Nebot, R. Tauler, J. Jaumot, Knowledge integration strategies for untargeted metabolomics based on MCRALS analysis of CE-MS and LC-MS data, Anal. Chim. Acta 978 (2017) 10-23.

[207] W. Zhang, K. Segers, D. Mangelings, A. Van Eeckhaut, T. Hankemeier, Y. Vander Heyden, R. Ramautar, Assessing the suitability of capillary electrophoresis-mass spectrometry for biomarker discovery in plasma-based metabolomics, Electrophoresis 40 (2019) 2309-2320.

[208] Y. Izadmanesh, E. Garreta-Lara, J.B. Ghasemi, S. Lacorte, V. Matamoros, R. Tauler, Chemometric analysis of comprehensive two dimensional gas chromatography-mass spectrometry metabolomics data, J. Chromatogr. A 1488 (2017) 113-125.

[209] M. Navarro-Reig, J. Jaumot, R. Tauler, An untargeted lipidomic strategy combining comprehensive two-dimensional liquid chromatography and chemometric analysis, J. Chromatogr. A 1568 (2018) 80-90.

[210] G.G. Siano, I.S. Pérez, M.D.G. García, M.M. Galera, H.C. Goicoechea, Multivariate curve resolution modeling of liquid chromatography-mass spectrometry data in a comparative study of the different endogenous metabolites behavior in two tomato cultivars treated with carbofuran pesticide, Talanta 85 (2011) 264-275.

[211] M.N. Sheikholeslami, C. Gómez-Canela, L.P. Barron, C. Barata, M. Vosough, R. Tauler, Untargeted metabolomics changes on Gammarus pulex induced by Propranolol, Triclosan, and Nimesulide pharmaceutical drugs, Chemosphere (2020), 127479.

[212] F. Marini, B. Walczak, ANOVA-target projection (ANOVA-TP), in: S. Brown, R. Tauler, R. Walczak (Eds.), Comprehensive Chemometrics, Elsevier, Oxford, 2020, pp. 495-520. 
[213] M. Martin, B. Govaerts, LiMM-PCA: combining ASCA + and linear mixed models to analyse high-dimensional designed data, J. Chemometr. 34 (2020) e3232.

[214] T.K. Karakach, R. Knight, E.M. Lenz, M.R. Viant, J.A. Walter, Analysis of time course $1 \mathrm{H}$ NMR metabolomics data by multivariate curve resolution, Magn. Reson. Chem. 47 (S1) (2009) S105-S117.

[215] F. Puig-Castellví, I. Alfonso, B. Piña, R. Tauler, 1 H NMR metabolomic study of auxotrophic starvation in yeast using multivariate curve resolutionalternating least squares for pathway analysis, Sci. Rep. 6 (2016), 30982.

[216] H. Motegi, Y. Tsuboi, A. Saga, T. Kagami, M. Inoue, H. Toki, O. Minowa, T. Noda, J. Kikuchi, Identification of reliable components in multivariate curve resolution-alternating least squares (MCR-ALS): a data-driven approach across metabolic processes, Sci. Rep. 5 (2015) 15710.

[217] V. Olmos, M. Marro, P. Loza-Alvarez, D. Raldúa, E. Prats, B. Piña, R. Tauler, A. de Juan, Assessment of tissue-specific multifactor effects in environmental-omics studies of heterogeneous biological samples: combining hyperspectral image information and chemometrics, Talanta 194 (2019) 390-398.

[218] C. Fernandes, E. Figueira, R. Tauler, C. Bedia, Exposure to chlorpyrifos induces morphometric, biochemical and lipidomic alterations in green beans (Phaseolus vulgaris), Ecotoxicol. Environ. Saf. 156 (2018) 25-33.

[219] Data Analysis for Omic Sciences, Methods and applications, in: J. Jaumot, C. Bedia, R. Tauler (Eds.), Comprehensive Analytical Chemistry vol. 82, Elsevier, 2018.

[220] J. Jaumot, R. Gargallo, A. de Juan, R. Tauler, A graphical user-friendly interface for MCR-ALS: a new tool for multivariate curve resolution in MATLAB, Chemometr. Intell. Lab. Syst. 76 (2005) (2005) 101-110.

[221] A.C. Olivieri, H.L. Wu, R.Q. Yu, MVC3: a MATLAB graphical interface toolbox for third-order multivariate calibration, Chemometr. Intell. Lab. Syst. 116 (2012) 9-16.

[222] A.C. Olivieri, G.M. Escandar, Practical Three-Way Calibration, Elsevier, Waltham, EEUU, 2014.

[223] C.H. Camp Jr., pyMCR: multivariate curve resolution in Python, J. Res. Nat. Inst. Standards Technol. 124 (2019). Art. 124018.

[224] M. Jalali-Heravi, H. Parastar, M. Kamalzadeh, R. Tauler, J. Jaumot, J. MCRC software: a tool for chemometric analysis of two-way chromatographic data, Chemom. Intell. Lab. Sys. 104 (2010) 155-171.

[225] J. Felten, H. Hall, J. Jaumot, R. Tauler, A. de Juan, A. Gorzsás, Vibrational spectroscopic image analysis of biological material using multivariate curve resolution-alternating least squares (MCR-ALS), Nat. Prot. 10 (2015) $217-240$.

[226] N. Mobaraki, J.M. Amigo, HYPER-Tools. A graphical user-friendly interface for hyperspectral image analysis, Chemom. Intell. Lab. Sys. 172 (2018) 174-187.

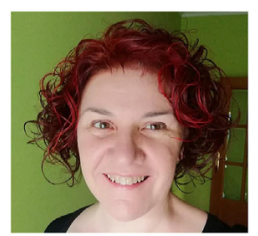

Anna de Juan Anna de Juan is an associate professor at the faculty of Chemistry of the Universitat de Barcelona. Her research focuses on the theoretical development of Multivariate Curve Resolution (MCR) and application to process analysis, hyperspectral image analysis and general analytical and bioanalytical problems. She has published more than 140 works and has given more than 200 presentations in international conferences. She received the 4th Chemometrics Elsevier Award in 2004 and the Kowalski Prize from J. of Chemometrics to the best applied paper in 2009. She serves in the Editorial Advisory Board of Chemometrics and Intelligent Laboratory Systems since 2002 and of Analytica Chimica Acta since 2006.

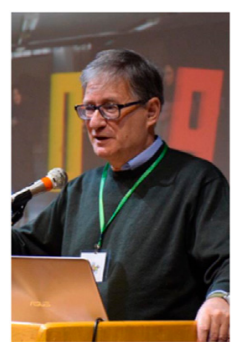

Romà Tauler (Barcelona, Spain, 1955) is research professor at IDAEA-CSIC. He obtained his doctorate in Analytical Chemistry at the University of Barcelona in 1977. Professor of the University of Barcelona 1978-2002. CSIC Professor since 2003. Chief Editor of J. of Chemometrics and Intelligent Laboratory Systems and of the Comprehensive Chemometrics Major Reference Work. Award for Achievements in Chemometrics, Eastern Analytical Symposium. 2009 Kowalski Prize from J. of Chemometrics, Wiley, 2009. President of the Catalan Chemistry Society, 2008-2013. Recipient of the EuERC Advanced Grant award Nr. 3203372013-2018. He has published more than 430 research publications and 60 book chapters, and has an h-index 63 . His main research field is Chemometrics and its applications to Environmental Chemistry, Omic Sciences, and Bioanalytical Chemistry.http://www.idaea.csic.es/http://www.ch4eo.infohttp:/ www.cid.csic.es/homes/rtaqam/http://mcrals.wordpress. com/download/mcr-als-toolbox/ 